This item was submitted to Loughborough's Research Repository by the author.

Items in Figshare are protected by copyright, with all rights reserved, unless otherwise indicated.

\title{
Aligned and misaligned contacts of rollers to races in elastohydrodynamic finite line conjunctions
}

PLEASE CITE THE PUBLISHED VERSION

PUBLISHER

Professional Engineering Publishing / @ IMECHE

VERSION

VoR (Version of Record)

LICENCE

CC BY-NC-ND 4.0

\section{REPOSITORY RECORD}

Kushwaha, M., Homer Rahnejat, and Ramsey Gohar. 2019. "Aligned and Misaligned Contacts of Rollers to Races in Elastohydrodynamic Finite Line Conjunctions". figshare. https://hdl.handle.net/2134/4771. 
This item was submitted to Loughborough's Institutional Repository (https://dspace.lboro.ac.uk/) by the author and is made available under the following Creative Commons Licence conditions.

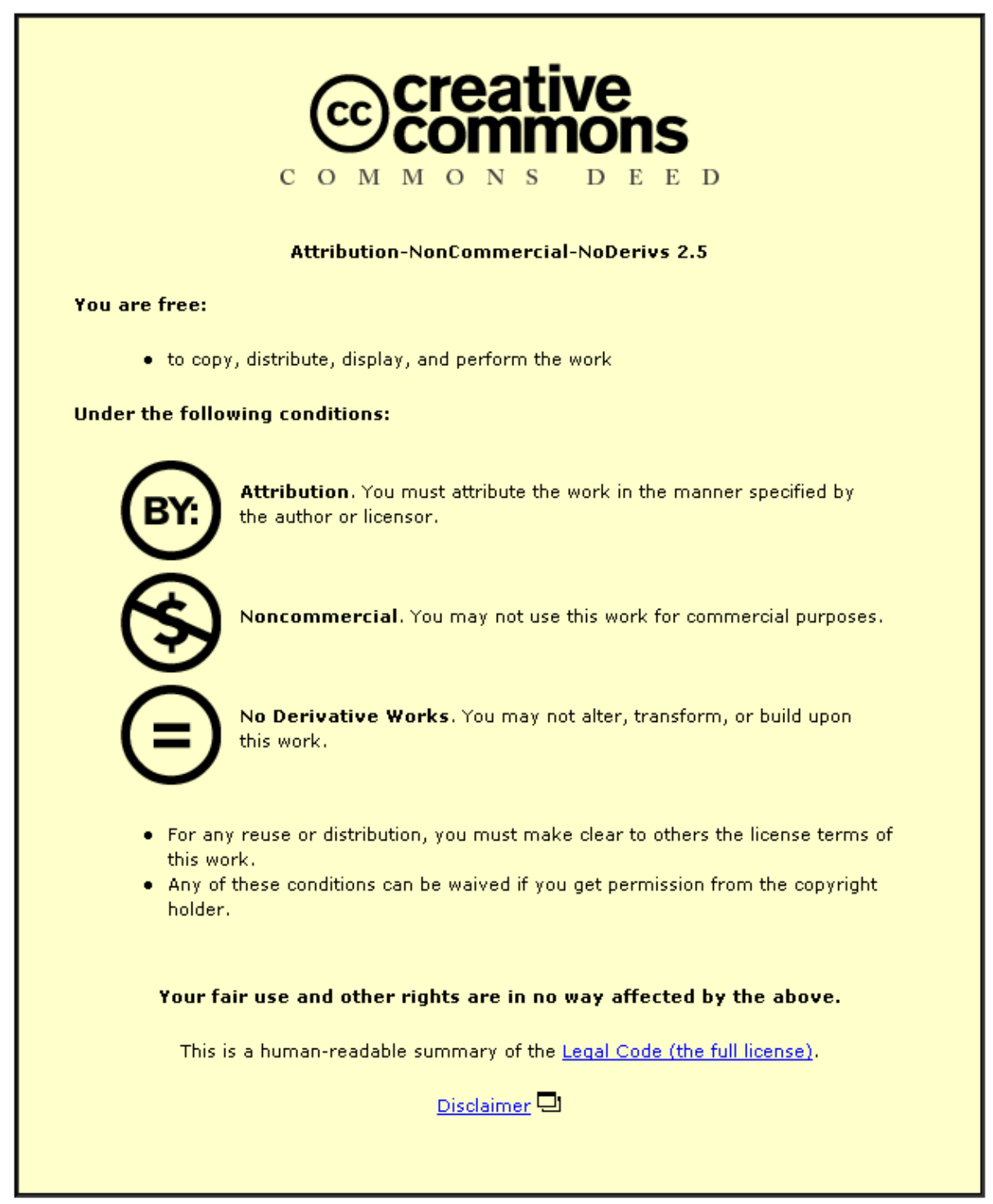

For the full text of this licence, please go to: http://creativecommons.org/licenses/by-nc-nd/2.5/ 


\title{
Aligned and misaligned contacts of rollers to races in elastohydrodynamic finite line conjunctions
}

\author{
M Kushwaha ${ }^{1}$, H Rahnejat ${ }^{1 *}$ and $\mathbf{R}$ Gohar $^{2}$ \\ ${ }^{1}$ Wolfson School of Mechanical and Manufacturing Engineering, Loughborough University, Loughborough, \\ Leicestershire, UK \\ ${ }^{2}$ Mechanical Engineering Department, Imperial College of Science, Technology and Medicine, University of London, \\ $\mathrm{UK}$
}

\begin{abstract}
The paper provides a solution for finite line concentrated contact of a roller-to-race under aligned and misaligned conditions. The lubricated contact conjunction is subject to an elastohydrodynamic regime of lubrication under isothermal conditions. Of particular interest are the edge stress discontinuities, represented by large secondary pressure spikes at the side constriction and to the rear exit in the contact domain. These pressure 'pips' are considerably larger in magnitude than those occurring in the central exit of the contact. The presence of pressure peaks inhibits the flow of lubricant in their vicinity, causing islands of minimum lubricant film thickness at the sides of the contact, referred to as the end closure films. The paper shows that the film shape and pressure distribution at the extremities of a finite line contact are not revealed by the traditional line contact solutions usually undertaken. The flow pattern becomes more complex with roller misalignment and the edge effects described are exacerbated. The paper provides the first ever solution of misaligned roller-to-race contact for moderate to high loaded elastohydrodynamic conjunctions. The numerical predictions conform well with both experimental and numerical findings of others for the cases where similar work has been reported.
\end{abstract}

Keywords: concentrated finite line contact, elastohydrodynamic lubrication, aligned and misaligned contacts

\section{INTRODUCTION}

Rolling element bearings are usually employed to support rotating shafts and are often subjected to combinations of radial and axial forces, as well as moments. For example, when a shaft is supported by a pair of non-self-aligning bearings at its ends, the bearings generate reaction forces, induced by the shaft deflection. Under an ideal loading condition, where misalignment of the shaft does not occur, the load distribution on an aligned roller-to-race contact can be obtained. Harris [1] postulated that when a roller bearing is subjected to pure radial forces, the load is evenly distributed along its effective length. However, in practice, roller misalignment occurs and relationships between the roller load, moment, deflection and its angular misalignment are required to solve the problem.

The MS was received on 26 February 2002 and was accepted after revision for publication on 19 A ugust 2002.

* Corresponding author: Wolfson School of Mechanical and Manufacturing Engineering, Loughborough University, Loughborough, Leicestershire LE11 $3 T U$, UK.
Gohar [2] provided a three-dimensional solution for the elastostatic pressure distribution for a roller in a cold rolling process. He used overlapping isoceles triangles of equal base length to represent the elastostatic pressures along the roller length and assumed an elliptical pressure distribution in the transverse direction. Using the same method, Heydari and Gohar [3] and Johns and Gohar [4] obtained elastostatic pressure distributions and corresponding footprint shapes for both aligned and misaligned rollers in rolling element bearings under dry contact conditions. In their solutions, a flexible shaft supported by a number of rolling bearings was assumed in order to yield a statically determinate system.

Rahnejat and Gohar [5] further extended the works in references [3] and [4] for the case of tapered roller bearings. Hartnett [6] used a similar method to that of Rahnejat and Gohar [5] to analyse the elastostatic pressure distribution in rolling elements-to-races contacts under dry contact conditions. Other research workers, e.g. Kannel [7] and Zantapulos [8], have also reported similar methods for the evaluation of elastostatic pressures and footprint shapes.

These studies showed the existence of high-pressure regions at the contact extremities, caused by the 
discontinuous nature of the roller axial profile at its ends. In order to reduce the edge stresses, rollers are blended at their ends or along their lengths. High pressures in the central region were first predicted in a one-dimensional infinite line contact elastohydrodynamic conjunction between mating discs or meshing teeth under a steady state entraining condition, for example, by Dowson and Higginson [9] and experimentally found by Niemann and Gartner [10]. Using the optical interferometric method for a roller on a flat glass disc contact, Wymer and Cameron [11] showed that the absolute minimum lubricant film thickness forms on either side of the contact (with respect to the direction of entraining motion) and to the rear, towards the exit constriction. The confirmation for this was found by a full two-dimensional solution for the elastohydrodynamic finite line conjunction by Mostofi and Gohar [12]. They found good agreement with the aforementioned experimental results, as indeed did Park and Kim [13] in a more recent numerical solution. Both of these finite line solutions used finite differences to solve the Reynolds equation, with heavy overrelaxation and the use of Gaussian elimination. The latter provides a slightly more refined solution in terms of computation time, using a partial pivoting technique, which uses the symmetric properties of the contact with respect to the direction of entraining motion for aligned rollers.

The use of the Voghepol transformation and Gaussian elimination meant that comparisons with experimental findings could be carried out where the load and entraining speeds were relatively low. Furthermore, such solution methods did not lend themselves to the investigation of misaligned roller conditions, particularly where the loss of contact symmetry calls for an increased mesh density, even with the use of irregular mesh spacings, as in references [12] and [13]. Use of the low relaxation effective influence Newton-Raphson (EIN) method, together with the Gauss-Seidel iterative procedure highlighted by Ehret et al. [14] and JalaliVahid et al. [15] provides for more accurate, stable and rapid solutions to the problem, with higher mesh densities in addition.

Misalignment of a roller gives rise to combined entraining with a tilting motion. The rigid-body squeeze effect was included in the finite line contact elastohydrodynamic analysis of mating discs by Rahnejat [16], by extending the steady state solutions of reference [12] to a quasi-static analysis for given ratios of the velocity of a normal approach to that of the speed of entraining motion. However, the effect of misalignment was ignored, as well as the local contributions due to the elastic squeeze film effect. This paper attempts to overcome the shortcomings in the aforementioned analyses, by providing solutions that are applicable for a larger range of operating conditions, as well as allowing comparison with previously reported experimental findings.

\section{METHOD OF FORMULATION}

To predict the pressure distribution and the corresponding lubricant film thickness, a simultaneous solution to the Reynolds equation and the elastic film shape in the conjunction formed by the contiguous bodies in contact is needed.

\subsection{Reynolds equation}

The dimensionless form of the Reynolds equation for a finite line contact of a roller against a flat can be written as

$$
\begin{aligned}
& \frac{\partial}{\partial \bar{x}}\left(\frac{\bar{\rho} \bar{h}^{3}}{\bar{\eta}} \frac{\partial \bar{P}}{\partial \bar{x}}\right)+\frac{b^{2}}{a^{2}} \frac{\partial}{\partial \bar{y}}\left(\frac{\bar{\rho} \bar{h}^{3}}{\bar{\eta}} \frac{\partial \bar{P}}{\partial \bar{y}}\right) \\
& \quad=12\left[\frac{u_{\mathrm{AV} \eta_{0} R^{2}}}{b^{3} P_{\mathrm{h}}} \frac{\partial}{\partial \bar{x}}(\bar{\rho} \bar{h})+\frac{\eta_{0} R^{2}}{b^{2} P_{\mathrm{h}}} \frac{\partial}{\partial t}(\bar{\rho} \bar{h})\right]
\end{aligned}
$$

where $\bar{x}$ denotes the direction of entraining motion. Note that $b$ is the half-width of the rectangular contact as shown in Fig. 1. The ultimate term in the equation can be simplified as

$$
\begin{aligned}
& 12 \frac{\eta_{0} R^{2}}{b^{2} P_{\mathrm{h}}} \frac{\partial}{\partial t}\left(\overline { \rho } _ { ( i , j ) } \left\lceil\left(\overline { h } _ { ( i , j ) } \left\lceil=12 \frac{\eta_{0} R^{2}}{b^{2} P_{\mathrm{h}}}\left(\bar{\rho}_{(i, j)}\right) \frac{\partial \bar{h}_{(i, j)}}{\partial t}\right.\right.\right.\right. \\
& \text { since } \frac{\partial \bar{\rho}_{(i, j)}}{\partial t} \approx 0
\end{aligned}
$$

Since a quasi-static analysis is carried out, no film history is available for the determination of the elastic body squeeze film action as defined by the term $\partial \bar{h}_{(i, j)} / \partial t$. Therefore, this term is ignored in the analysis.

The bulk density of the fluid is assumed to remain largely unaltered in a small interval of time, as the pressure distribution remains the same under instantaneous quasi-static conditions, studied here. To solve the Reynolds equation, isothermal conditions were assumed and a rheological model was used to describe the lubricant density and viscosity variations with pressure.

\subsection{Lubricant viscosity}

Roelands [17] derived an expression for the lubricant viscosity variation with pressure for mineral oils under isothermal conditions as

$$
\bar{\eta}=\exp \left\{\left(\ln \eta_{0}+9.67\right)\left[-1+\left(1+\left.\Im \bar{P} P_{\mathrm{h}}\right|^{\Re}\right]\right\}\right.
$$

where $\Im=5.1 \times 10^{-9}$, a constant with units of $\mathrm{m}^{2} / \mathrm{N}$. It must be noted that in the Roelands equation, the lubricant viscosity is defined using three different parameters [i.e. the atmospheric viscosity $\left(\eta_{0}\right)$, the asymptotic isoviscous pressures $\left(P_{\text {iv.as }}\right)$ and the pressure-viscosity index $(\Re=0.67)]$. 


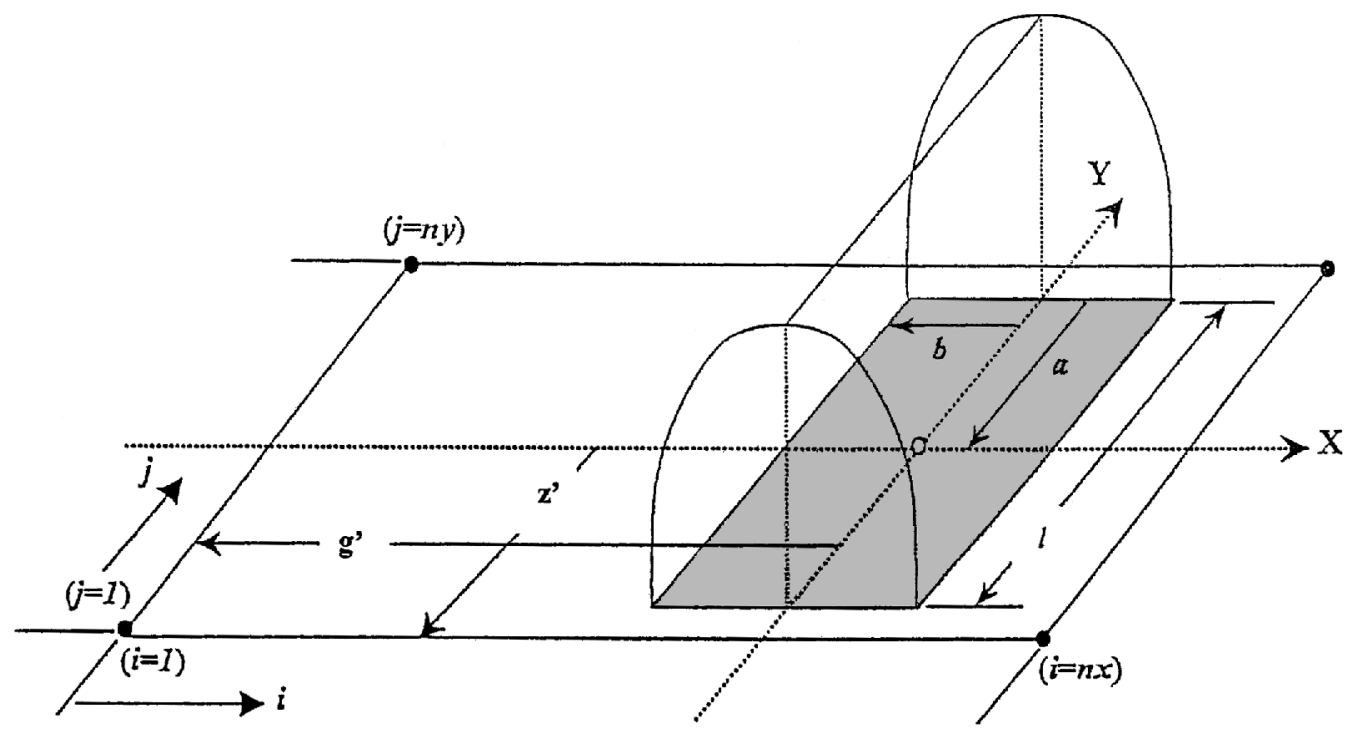

Fig. 1 Hertzian pressure distribution for a finite line contact

\subsection{Lubricant density}

The variation in density of the lubricant with pressure is defined by Dowson and Higginson [9] as

$$
\bar{\rho}=1+\frac{\alpha \bar{P} P_{\mathrm{h}}}{1+\beta \bar{P} P_{\mathrm{h}}}
$$

where $\alpha$ and $\beta$ are constants, dependent upon the properties of the fluid. The values used in the current analysis are $5.83 \times 10^{-10}$ and $1.68 \times 10^{-9}$ respectively, which are based on mineral oils.

\subsection{The elastic film shape}

When an analysis of a line contact is carried out, the axial contact dimension is usually considered to be infinite. The reason behind this assumption is that under lubricated conditions, the lubricant side flow (i.e. the side leakage) is ignored, thus making the problem one dimensional. This, however, does not conform to practical situations, as no element in contact has an infinite dimension. Therefore, the contact width, transverse to the flow direction, is made finite. Since an equivalent system is used, where the roller is considered to be in contact with a flat elastic half-space, only the deformation of the elastic surface is considered. Assuming the undeformed profile of the roller to be parabolic, the lubricant film thickness at any location within the contact domain can be expressed as (see Fig. 2)

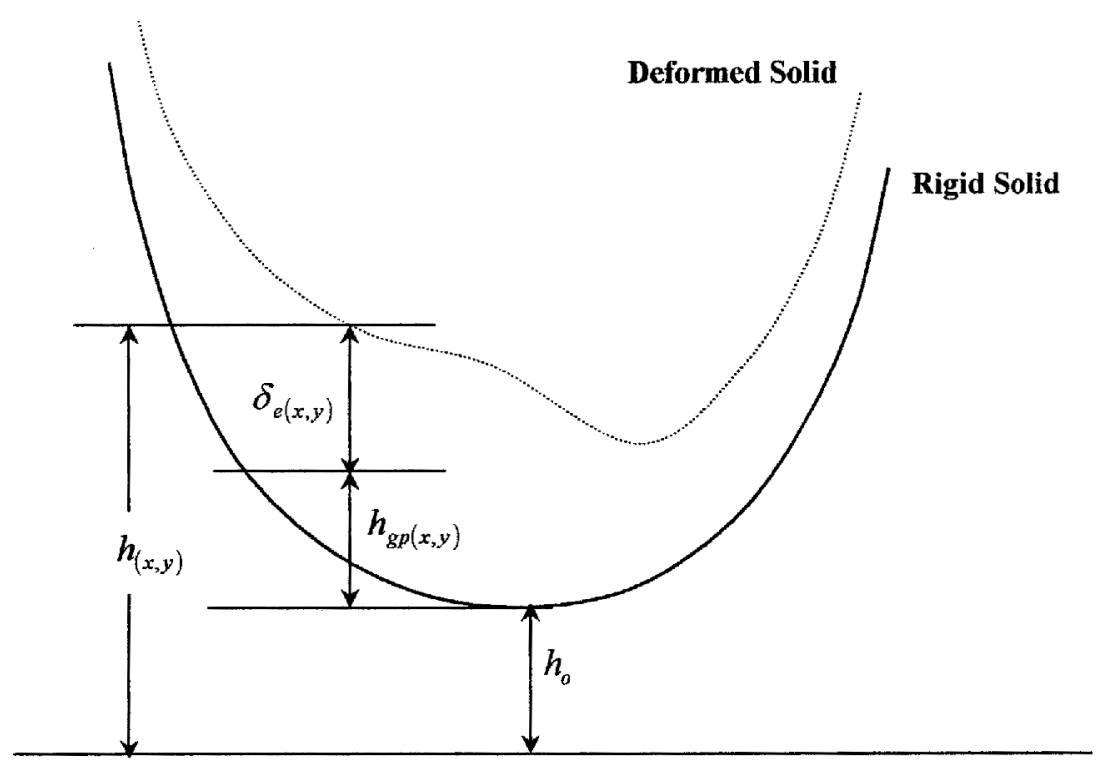

Fig. 2 Components defining the elastic film shape equation (5) 


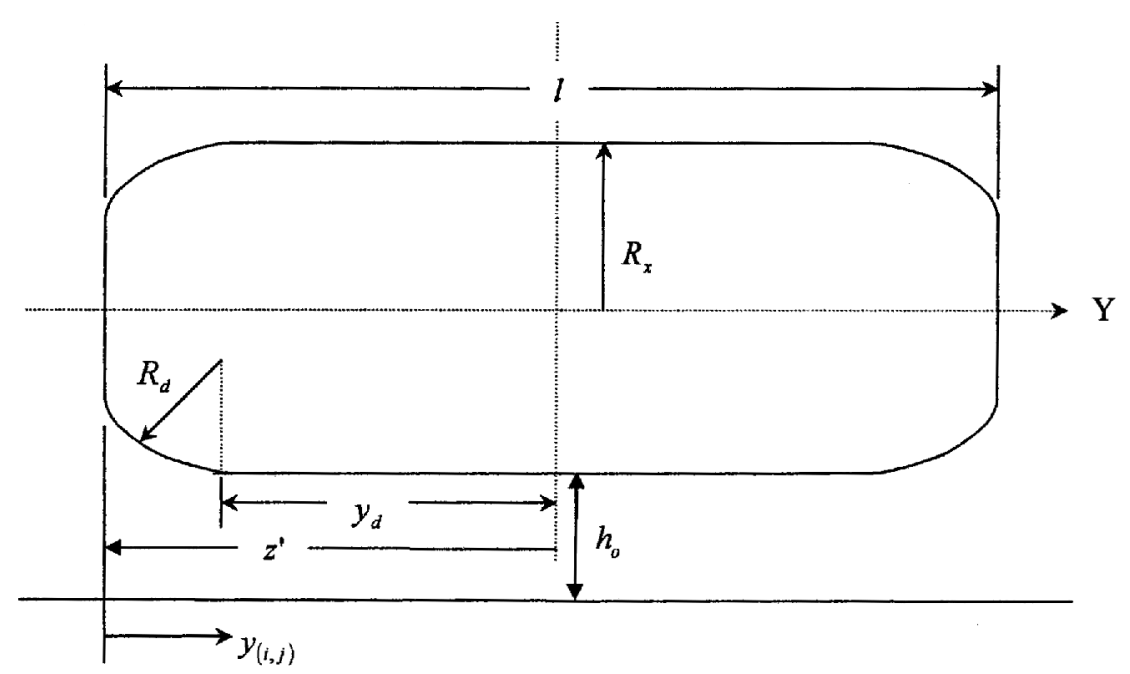

Fig. 3 Roller profile in the $Y$ direction (axial) $\left(R_{\mathrm{d}}=\right.$ dub-off radius)

$$
h_{(x, y)}=h_{0}+h_{\mathrm{gp}(x, y)}+\delta_{\mathrm{e}(x, y)}
$$

As the roller is edge-blended in order to reduce edge stress concentrations, the alteration in its axial undeformed profile must be taken into account when evaluating $h_{\mathrm{gp}(x, y)}$ (see Fig. 3).

By the principle of superposition, the total elastic deflection at node $(k, l)$ can be formulated as [18]

$$
\delta_{\mathrm{e}(k, l)}=\frac{2}{\pi E^{\prime}} \sum_{j=1}^{n x} \sum_{i=1}^{n y} p_{i, j} D_{m, n}
$$

where

$$
m=|k-i+1| \quad \text { and } \quad n=|l-j+1|
$$

and

$$
\begin{aligned}
& D_{m, n}=\left(y-\frac{\Delta y}{2}\right) \ln \left[\frac{\left(x-\frac{\Delta x}{2}\right)+\sqrt{\left(y-\frac{\Delta y}{2}\right)^{2}+\left(x-\frac{\Delta x}{2}\right)^{2}}}{\left.\left(x+\frac{\Delta x}{2}\right)+\sqrt{\left(y-\frac{\Delta y}{2}\right)^{2}+\left(x+\frac{\Delta x}{2}\right)^{2}}\right]}\right. \\
&+\left(y+\frac{\Delta y}{2}\right) \ln \left[\frac{\left(x+\frac{\Delta x}{2}\right)+\sqrt{\left(y+\frac{\Delta y}{2}\right)^{2}+\left(x+\frac{\Delta x}{2}\right)^{2}}}{\left(x-\frac{\Delta x}{2}\right)+\sqrt{\left(y+\frac{\Delta y}{2}\right)^{2}+\left(x-\frac{\Delta x}{2}\right)^{2}}}\right] \\
&+\left(x+\frac{\Delta x}{2}\right) \ln \left[\frac{\left(y+\frac{\Delta y}{2}\right)+\sqrt{\left(y+\frac{\Delta y}{2}\right)^{2}+\left(x+\frac{\Delta x}{2}\right)^{2}}}{\left(y-\frac{\Delta y}{2}\right)+\sqrt{\left(y-\frac{\Delta y}{2}\right)^{2}+\left(x+\frac{\Delta x}{2}\right)^{2}}}\right] \\
&+\left(x-\frac{\Delta x}{2}\right) \ln \left[\frac{\left(y-\frac{\Delta y}{2}\right)+\sqrt{\left(y-\frac{\Delta y}{2}\right)^{2}+\left(x-\frac{\Delta x}{2}\right)^{2}}}{\left(y+\frac{\Delta y}{2}\right)+\sqrt{\left(y+\frac{\Delta y}{2}\right)^{2}+\left(x-\frac{\Delta x}{2}\right)^{2}}}\right]
\end{aligned}
$$

Using parabolic lateral and axially blended undeformed profiles of the roller (see Fig. 3) and the nondimensionalizing equation (5), the elastic film shape becomes

$$
\begin{aligned}
\bar{h}_{(\bar{x}, \bar{y})}= & \bar{h}_{0} \\
& +\left\{\frac{\left(\bar{x}_{i, j}-\left.\bar{g}^{\prime}\right|^{2}\right.}{2}+\frac{\left[\bar{y}_{i, j}-\left(\bar{z}^{\prime}-\bar{y}_{\mathrm{d}}\right)\right]^{2}}{2 R_{\mathrm{d}}}\left(\frac{a^{2} R}{b^{2}}\right)\right\} \\
& +\bar{\delta}_{\mathrm{e}(\bar{x}, \bar{y})}
\end{aligned}
$$

Note that $\bar{z}^{\prime}$ and $\bar{g}^{\prime}$ are coordinate shifts in the $Y$ and $X$ directions respectively from the centre of the contact to the origin of the computation domain for the description of the roller profile (see Fig. 1). The term in the brace in equation (9) provides the blended end profile of the roller (see Fig. 3), with the mid-region having a zero profile. Symmetry is employed to set the profile at the axial extremities.

\subsection{Load balance}

In non-dimensional form, the applied load is defined by its Hertzian equivalent pressure distribution as

$$
W=\frac{\pi P_{\mathrm{h}} b l}{2}
$$

where $l=2 a$. The normal load carried by the generated elastohydrodynamic pressures over the contact area can be defined as

$$
W=\int_{A} \bar{P} P_{\mathrm{h}} \mathrm{d} \bar{x} b \mathrm{~d} \bar{y} a
$$


Equating the above loads and after some simplification,

$$
\bar{W}=\int_{A} \bar{P} \mathrm{~d} \bar{x} \mathrm{~d} \bar{y}=\pi
$$

\section{METHOD OF SOLUTION}

\subsection{Computation domain}

Once the dimensions of the elastostatic footprint for a particular load and geometry are calculated, a domain that is generally larger than the contact area is identified. Assuming the entraining velocity vector $\boldsymbol{u}_{\mathrm{AV}}=\frac{1}{2}\left(u_{1}+\right.$ $u_{2}$ ) to be in the $X$ direction in Fig. 4, a regular mesh with finite nodal points is constructed over the rectangular computational zone. As shown in figure, the size of this zone is such that it is about 4.5 to 5.0 times the elastostatic footprint half-width in the inlet region of the lubricated conjunction, and about 1.5 to 1.6 times that at the outlet.

Along the axial length of the roller (i.e. in the $Y$ direction), the zone is extended at both sides to about 4-8 per cent of the elastostatic footprint half-length. The percentage extension of the boundary can be varied to obtain the best starting position of the pressure curve in the axial direction. The dimensions shown in the figure correspond to approximate fully flooded conditions. It must be noted that if the entraining velocity is very high, then the inlet boundary must be increased accordingly to accommodate for the elongated inlet trail.

For the solution of the finite line elastohydrodynamic lubrication (EHL) problem, a grid of 64 elements in the entraining direction and 162 elements in the axial direction is employed. These can, however, be increased to obtain a finer mesh, which in turn may result in more accurate solutions.

\subsection{Solution of the Reynolds equation}

The Reynolds equation is discretized at every node in the computation domain and is rewritten in the following form:

$$
\sum_{k=2}^{n x-1} \sum_{l=2}^{n y-1}\left[\bar{J}_{(i j, k l)}\right] \Delta \bar{P}_{(k, l)}=-f_{(i, j)}^{\mathrm{R}}
$$

The left-hand side differentials form the Jacobian terms $\left(\partial f_{(i, j)}^{\mathrm{J}} / \partial \bar{P}_{(k, l)}\right)$ and the right-hand term forms the residual function $f^{\mathrm{R}}$ in central difference discretization, this being the Reynolds equation itself. The Jacobian terms are given in backward differences. The residual

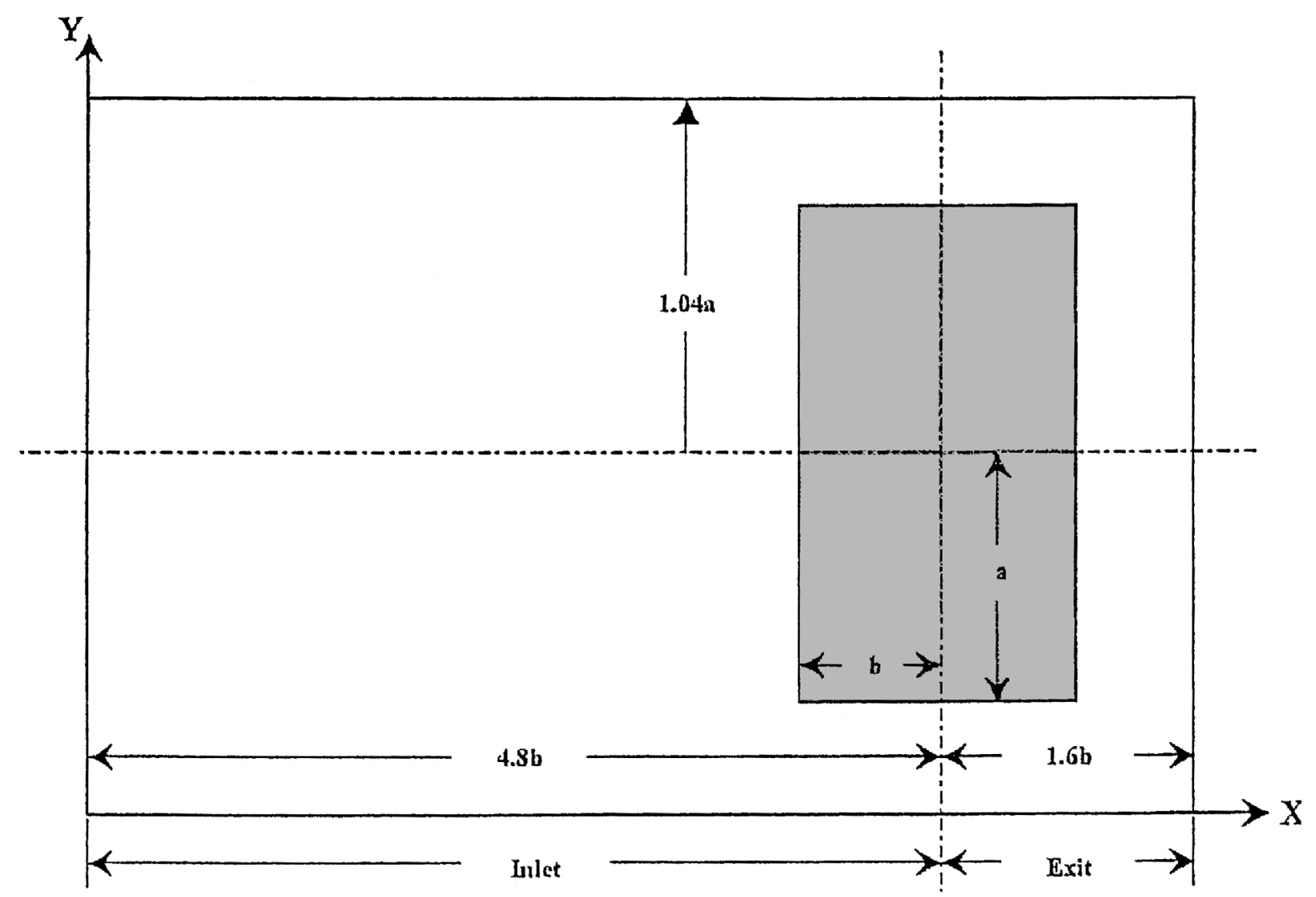

Fig. 4 Dimensions of the computational grid 
term is, therefore,

$$
\begin{aligned}
& f^{\mathrm{R}}=\frac{1}{2 \Delta \bar{x}^{2}}\{ {\left[\left(\frac{\bar{\rho} \bar{h}^{3}}{\bar{\eta}}\right)_{(i+1, j)}+\left(\frac{\bar{\rho} \bar{h}^{3}}{\bar{\eta}}\right)_{(i, j)}\right]_{P_{(i+1, j)}-}\left[\left(\frac{\bar{\rho} \bar{h}^{3}}{\bar{\eta}}\right)_{(i+1, j)}+2\left(\frac{\bar{\rho} \bar{h}^{3}}{\bar{\eta}}\right)_{(i, j)}+\left(\frac{\bar{\rho} \bar{h}^{3}}{\bar{\eta}}\right)_{(i-1, j)}\right] \bar{P}_{(i, j)} } \\
&+ {\left.\left[\left(\frac{\bar{\rho} \bar{h}^{3}}{\bar{\eta}}\right)_{(i, j)}+\left(\frac{\bar{\rho} \bar{h}^{3}}{\bar{\eta}}\right)_{(i-1, j)}\right] \bar{P}_{(i-1, j)}\right\} } \\
&+\frac{b^{2}}{2 \Delta \bar{y}^{2} a^{2}}\left\{\left[\left(\frac{\bar{\rho} h^{3}}{\bar{\eta}}\right)_{(i, j+1)}+\left(\frac{\bar{\rho} \bar{h}^{3}}{\bar{\eta}}\right)_{(i, j)}\right] \bar{P}_{(i, j+1)}-\left[\left(\frac{\bar{\rho} \bar{h}^{3}}{\bar{\eta}}\right)_{(i, j+1)}+2\left(\frac{\bar{\rho} \bar{h}^{3}}{\bar{\eta}}\right)_{(i, j)}+\left(\frac{\bar{\rho} \bar{h}^{3}}{\bar{\eta}}\right)_{(i, j-1)}\right] \bar{P}_{(i, j)}\right. \\
&\left.+\left[\left(\frac{\bar{\rho}^{3}}{\bar{\eta}}\right)_{(i, j)}+\left(\frac{\bar{\rho}^{3}}{\bar{\eta}}\right)_{(i, j-1)}\right] \bar{P}_{(i, j-1)}\right\}-6 \frac{u_{\mathrm{AV} \eta_{0} R^{2}}}{b^{3} P_{\mathrm{h}}}\left[\frac{\left(\bar{\rho} \bar{h} \Gamma_{(i+1, j)}-\left(\bar{\rho} \bar{h} \Gamma_{(i-1, j)}\right.\right.}{\Delta \bar{x}}\right]
\end{aligned}
$$

The Reynolds equation for the Jacobian terms is discretized, using the same method as that given above, except for the right-hand side of the equation, where the Couette flow terms are discretized, using the standard backward differencing scheme in order to achieve a better degree of numerical stability. Hence, the flow terms can be written as

$$
12 \frac{u_{\mathrm{AV}} \eta_{0} R^{2}}{b^{3} P_{\mathrm{h}}}\left[\frac{\left(\overline { \rho } \overline { h } \left\lceil_{(i, j)}-\left(\overline { \rho } \overline { h } \left\lceil_{(i-1, j)}\right.\right.\right.\right.}{\Delta \bar{x}}\right]
$$

Therefore, the Reynolds equation for the evaluation of the Jacobian term $f^{\mathrm{J}}$ is similar to equation (14) with the Couette term replaced by equation (15).

\subsection{Boundary conditions}

The boundary conditions employed are:

1. At the boundaries of the rectangular computational zone the pressures are zero. Thus, for a mesh of 65 by 163 ,

$$
\bar{P}_{(i, 1)}=\bar{P}_{(65, j)}=\bar{P}_{(i, 163)}=\bar{P}_{(1, j)}=0
$$

2. To avoid the generation of negative pressures in the fluid film, at the cavitation boundary, the Reynolds condition

$$
\bar{P}=\frac{\partial \bar{P}}{\partial \bar{x}}=\frac{\partial \bar{P}}{\partial \bar{y}}=0
$$

is employed. Numerically, $\bar{P}=0$ whenever the pressure is negative.

\subsection{Initial conditions}

The pressure distribution inside the elastostatic footprint for a particular load is initially assumed to be
Hertzian, while outside this area the pressure equates to zero.

\subsection{Convergence criteria}

Now the solution to equation (13) can be obtained, using an error tolerance with the Newton-Raphson method. If a solution obtained is within the limits of the required tolerance, then the numerical procedure is deemed to have converged. For the pressures to converge, the following criterion is employed:

$$
\frac{\sum_{i=1}^{n x} \sum_{j=1}^{n y}\left|\bar{P}_{(i, j)}^{n}-\bar{P}_{(i, j)}^{0}\right|}{\sum_{i=1}^{n x} \sum_{j=1}^{n y} \bar{P}_{(i, j)}^{n}} \leqslant \operatorname{Err}_{P}
$$

where the error tolerance for the pressure $\operatorname{Err}_{P}=0.0001$.

If convergence is not achieved, the pressures are updated as

$$
\bar{P}_{(i, j)}^{n}=\bar{P}_{(i, j)}^{0}+\lambda \Delta \bar{P}_{(i, j)}
$$

where $\lambda$ is the under-relaxation factor with the usual range $0.1-1.0$, depending on the type of problem at hand. Similarly, for the load to reach a converged solution, the criterion used is

$$
|\bar{W}-\pi| \leqslant \operatorname{Err}_{W}
$$

Here, the error tolerance for load convergence $\operatorname{Err}_{W}$ is set equal to 0.00005 . If the load has not converged, then the central film thickness is adjusted according to the evaluated unbalanced load, using the following relationship:

$$
\bar{H}_{0}^{n}=\bar{H}_{0}^{0}+\hbar(\bar{W}-\pi)
$$

where $\hbar$ is a damping coefficient used to reduce the sharp variations in the iterative values of the central oil-film 
thickness, and lies between 0.001 and 0.05 . Hence, if the evaluated load $\bar{W}$ is less than $\pi$, then the central film thickness is further reduced.

\section{MISALIGNED CONTACTS}

A certain amount of axial misalignment of the rollers is always known to exist in all applications of roller bearings due to moment loading conditions [19]. This is because, under equilibrium conditions, rolling element bearings already undergo some pre-load once the assembly is complete, either due to the static deflection of the shaft, resulting from the existing radial loads or due to settling of machine foundation, as explained by Johns [19]. Hence, the effect of misalignment should be included when the shaft experiences high radial loads under dynamic operating conditions.

An estimate of the maximum roller misalignment angle of the bearing can be obtained assuming elastostatic contacts. For zero radial clearance in a rolling bearing having $N_{\mathrm{R}}$ number of rollers and a total bearing radial load $F_{\mathrm{r}}$, the maximum load on the bottom roller is given by $\mathrm{H}$ arris $[\mathbf{2 0}]$ as

$$
W_{\max }=\frac{4.08 F_{\mathrm{r}}}{N_{\mathrm{R}}}
$$

An approximate relationship between the roller load and its deflection has been obtained by Lundberg [21] for the mutual approach of a finite roller and an elastic half-space as

$$
\delta_{0} \cong 3.81 \frac{W^{0.9}}{l^{0.8}}\left[\frac{2\left(1-v^{2}\right)}{\pi E}\right]^{0.9}
$$

which, for a steel-on-steel contact, equates to the following expression in Imperial units [21]:

$$
\delta_{0} \cong 4.36 \times 10^{-7} \frac{W^{0.9}}{l^{0.8}}
$$

Harris has approximated this expression to [20]

$$
\delta_{0}=K q
$$

where $q=W / l$.

Assume, as an approximation, that the roller can be considered as a series of laminae behaving independently (i.e. under a plane strain condition); the deflection at any point along its length can be stated as $[4,19]$

$$
\delta_{\mathrm{e}(y)}=\delta_{0}+\theta y
$$

Using the above equation, the following expression can be obtained:

$$
q_{(y)}=\frac{1}{K}\left(\delta_{0}+\theta y\right)
$$

The Harris theory can be used to determine the misalignment angle for any roller by observing that the moment load on any $i$ th roller is given as

$$
M_{i}=\int_{-1 / 2}^{1 / 2} q_{i} y \mathrm{~d} y
$$

If $\theta_{\max }$ is the misalignment angle for the bottom roller, using the above equations it follows that

$$
M_{\max }=\frac{\theta_{\max } l^{3}}{12 K}
$$

If now simple beam theory is used to represent a shaft supported symmetrically by a pair of rolling element bearings carrying a radial load of $W_{\text {sh }}$, it follows that

$$
\begin{aligned}
& F_{\mathrm{r}}=\frac{W_{\mathrm{sh}}}{2} \\
& M=\frac{W_{\mathrm{sh}} L}{8}-\frac{2 E I \varphi}{L}
\end{aligned}
$$

For elastic rollers and races $[4,19]$,

$$
\theta_{\max }=\frac{\varphi}{2}
$$

The moment distribution for a rolling element bearing with the top rollers being unloaded is given as

$$
M=\sum_{\psi=-\pi / 2}^{\pi / 2} M_{i} \cos \psi_{i}
$$

where $M_{i}=M_{\max } \cos \psi_{i}$. Therefore, upon substitution,

$$
M=M_{\max } \sum_{\psi_{i}=-\pi / 2}^{\pi / 2} \cos ^{2} \psi_{i}
$$

Using the above relations,

$$
\theta_{\max }=\frac{W_{\mathrm{sh}} L}{8\left\{\left[l^{3} /(12 K)\right]\left(\sum \cos ^{2} \psi_{i}\right)+(4 E I / L)\right\}}
$$

The left-hand term in the denominator of equation (32) is the end-fixing moment supplied by the bearing. Had the shaft been simply supported, Young's modulus in this term would have to be infinite. The term on the right-hand side of the denominator would then have given the shaft end slope. Equation (32) also shows that the misaligned bottom roller in a rolling element bearing is able to supply very little angular stiffness and also has a load tilt angle [4]. However, even this low angle can cause a considerable increase in the maximum value of the resulting EHL pressure distribution, as will be shown below. With values of $W_{\max }$ and $\theta_{\max }$ obtained by 


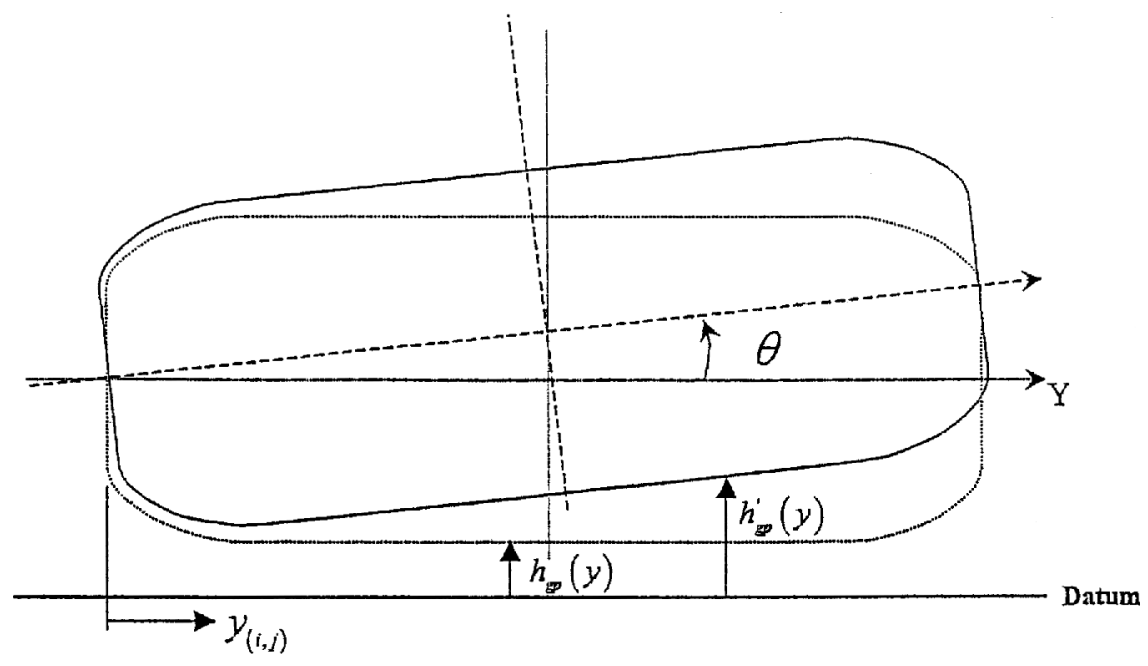

Fig. 5 Geometrical profile for the misaligned roller

the above calculations and the geometry of the roller to raceway contact being available, the elastohydrodynamic pressure distribution and the corresponding lubricant film thickness can be determined.

For a given misalignment angle, $\theta$, measured from one end of the roller in the $Y$ direction, the undeformed geometrical profile of the roller in the new position can be expressed as (see Fig. 5):

$$
h_{\mathrm{gp}}^{\prime}(y)=h_{\mathrm{gp}}(y)+y_{(i, j)} \tan \theta
$$

The primed term, representing the undeformed misaligned profile in the above expression, replaces the aligned roller profile in equation (5).

\section{RESULTS AND DISCUSSION}

The footprint shape for the finite line contact of a roller pressed into an elastic half-space has been found to be like a dogbone (or a dumbell) shape by a number of workers $[3,4,7,12,13,16,19,22,23]$. Under lubricated conditions, the oil-film contour has a similar shape. A guide contour map is shown in Fig. 6. This guide contour is used to refer to the various results presented in this section. The various cross-sections in the direction of entraining motion and in the transverse direction have been indicated on the figure. The sections 1-1 and 3-3 are through the central contact domain in the rolling and transverse directions, while the sections 2-2, 4-4 and 5-5 are through the side constriction (end closure).

This approach, discussed in Section 4, was employed by Johns and Gohar [4] to obtain the operating conditions on the highest loaded roller with a maximum misalignment angle (i.e. the bottom roller). In the current analysis, pressure distribution and correspond- ing film shape have been obtained under the same conditions as used by Johns and Gohar [4] for a dry elastostatic contact.

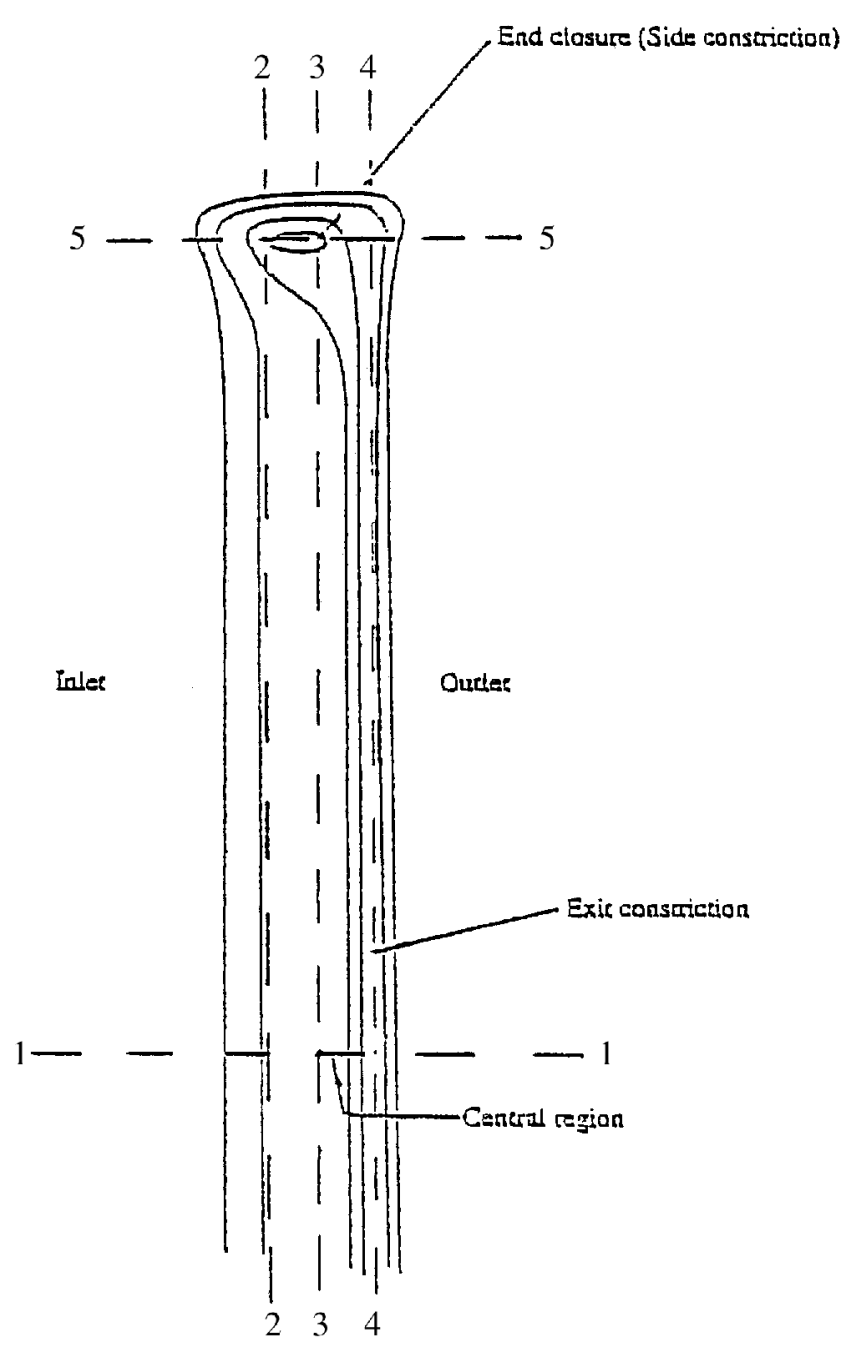

Fig. 6 Guide contour for finite line contacts 


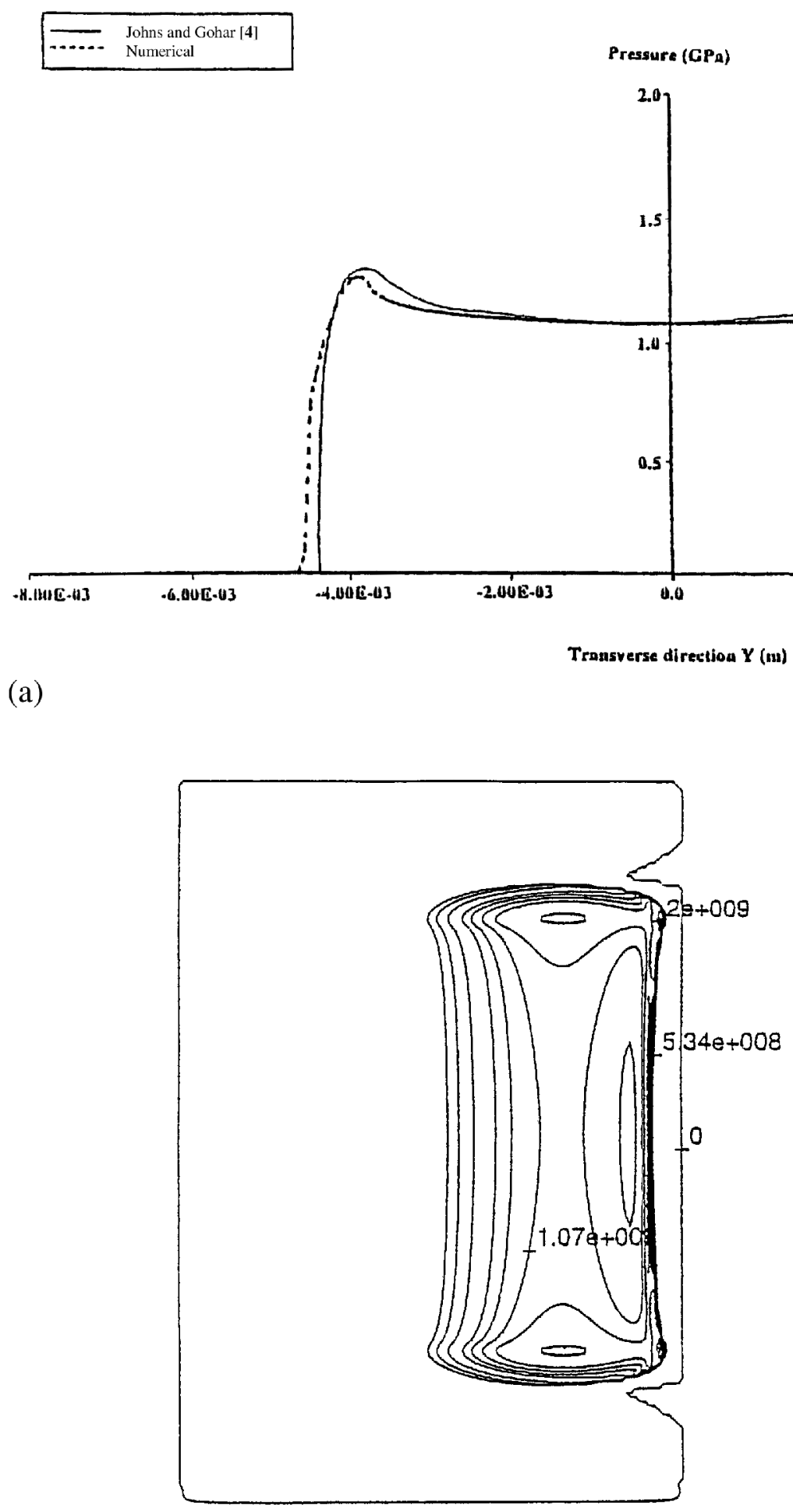

(b)

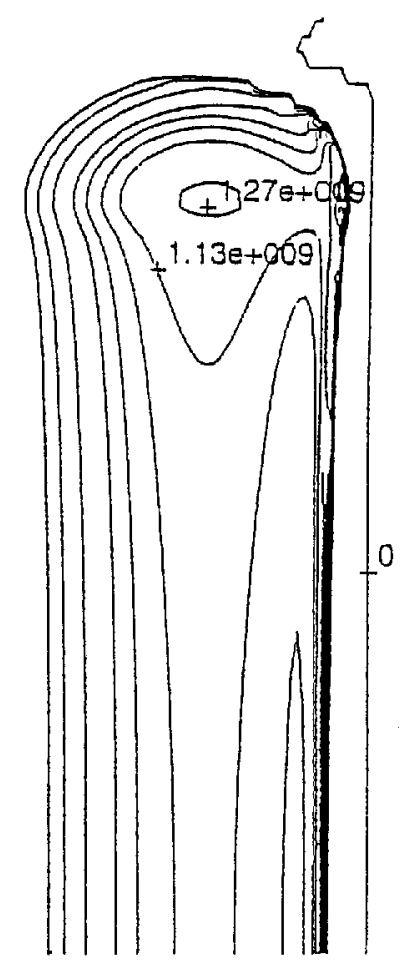

Fig. 7 (continued over)
Figure 7a shows two central pressure profiles in the transverse direction for an aligned roller with dub-off edges, under a load of $2256 \mathrm{~N}$. It can be observed that good agreement exists between both results. The pressure over the central flat region is found to be similar, with slightly reduced pressure spikes along this central contacting region. As expected, the contact length is extended slightly in the case of the lubricated 


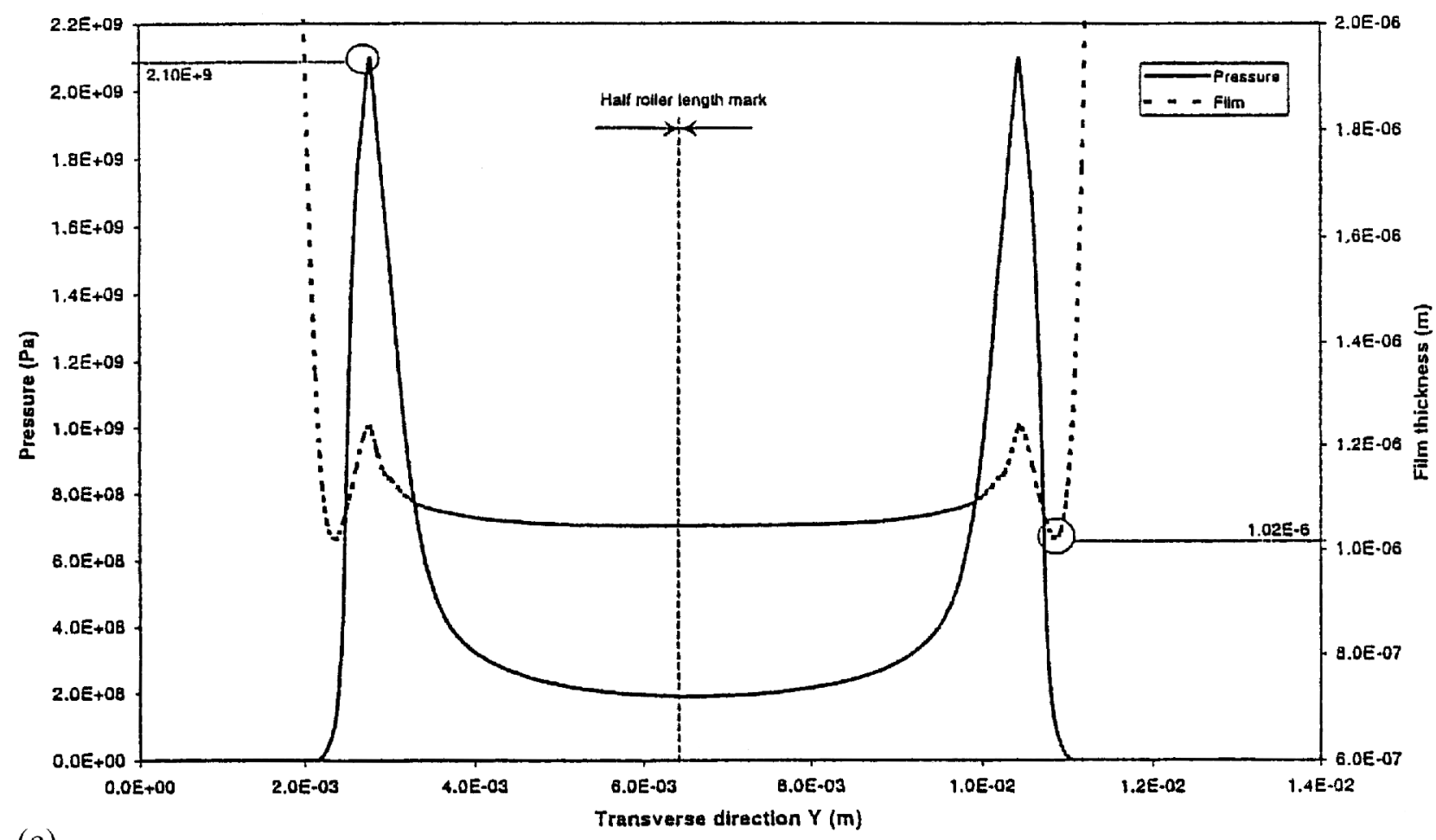

(c)

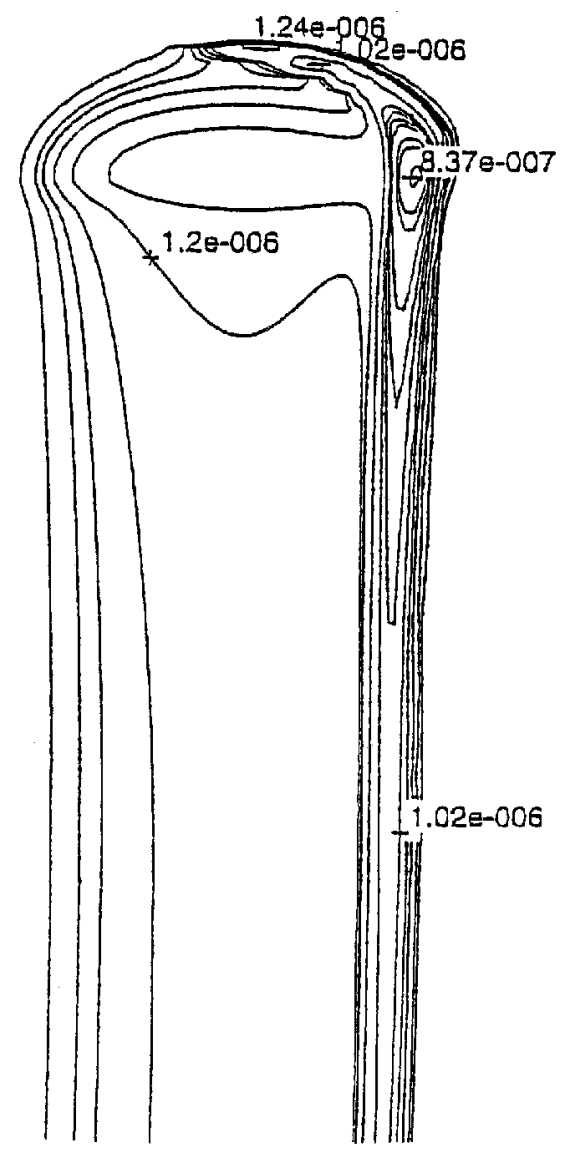

(d)

Fig. 7 (continued over) 


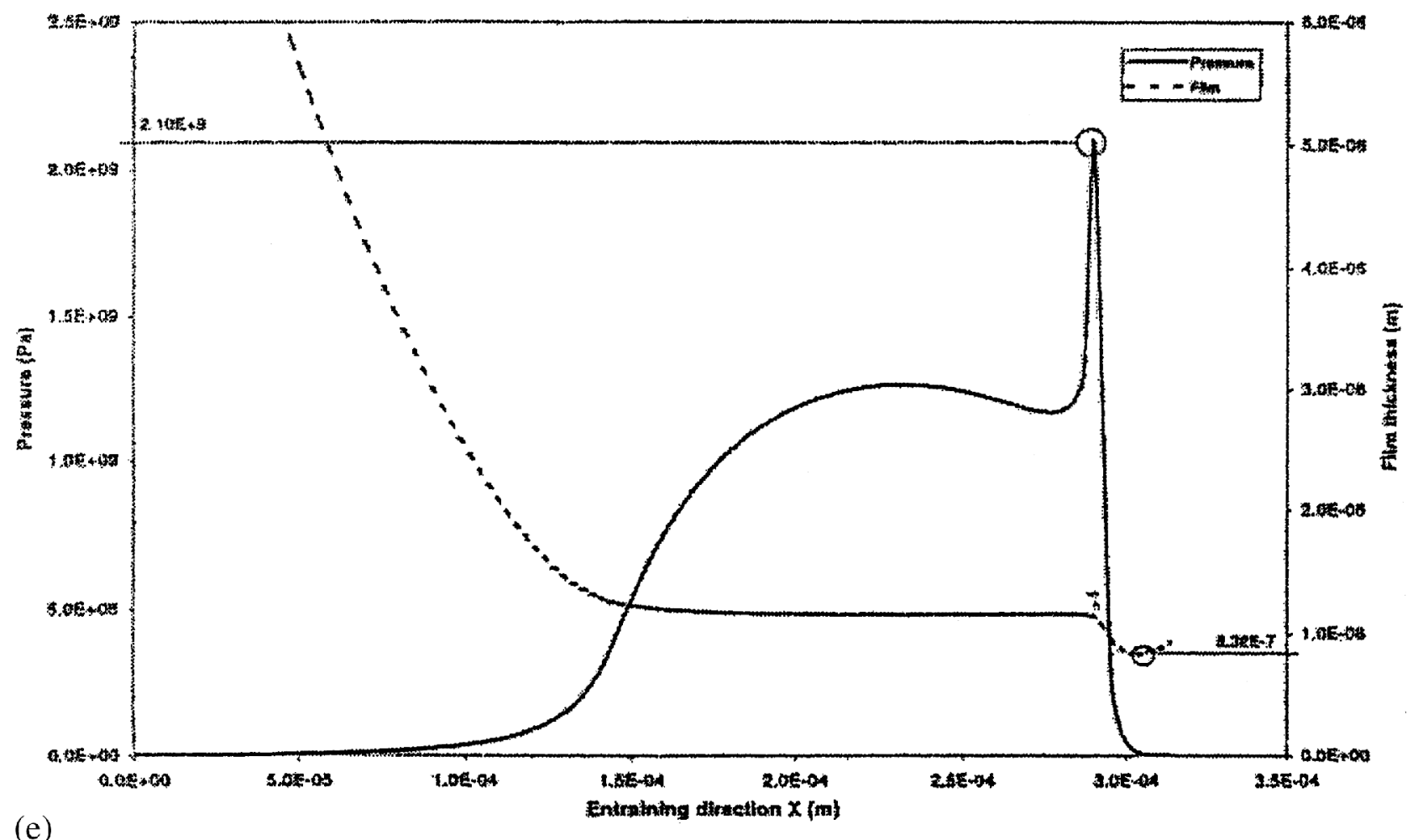

Fig. 7 (a) Central pressure profiles along section 3-3. (b) Pressure isobars for the lubricated contact (dimensional). (c) Axial pressure and corresponding film profiles along section 4-4. (d) Oil-film contour for the lubricated contact. (e) Pressure and corresponding film profiles along section 5-5

solution to include the side lobes. Figure $7 \mathrm{~b}$ shows the pressure isobars for the lubricated contact under the elastohydrodynamic regime of lubrication. It can be observed that the region of maximum pressures is at the side constriction, indicated on the inset to the figure, which has been enlarged in the vicinity of the side constriction. The maximum value of the pressure spike has been obtained as $2.1 \mathrm{GPa}$ at the side constriction and in the region of the rear exit. Figure $7 \mathrm{c}$ illustrates the pressure profile in the transverse direction through the side constriction (section 4-4 of the guide contour). The corresponding film thickness is also shown in the same figure. The minimum film thickness indicated along this section is $1.02 \mu \mathrm{m}$. The absolute minimum film thickness is along a section to the rear of section 4-4 and immediately prior to the exit constriction. Its value is $0.837 \mu \mathrm{m}$, as shown in the oil-film contour of Fig. $7 \mathrm{~d}$. The pressures are much lower here than those to the side of this exit constriction. This absolute minimum film thickness can be observed through section $5-5$ in the direction of entraining motion in Fig. 7e. The pressure profile along this section is also shown in the same figure, having the highest pressure element in the contact domain at $2.1 \mathrm{GPa}$. Returning to Fig. $7 \mathrm{a}$, it should be noted that the pressure spikes are much lower in value (i.e. approximately $1.3 \mathrm{GPa}$ ). The lower values of pressure and the correspondingly higher film thickness values along the central sections, both in the entraining and the lateral directions, when compared with the corresponding values at the side and the rear constrictions, indicate that the one-dimensional solution of the Reynolds equation in the case of roller to races, cam to follower or gear meshing teeth is inappropriate. Such actions would lead to underestimation of the value of contact pressures and overestimation of the value of film thickness. Therefore, neither fatigue life nor wear performance of contacting members in such concentrated contacts can be predicted accurately, unless a three-dimensional finite line analysis is carried out.

Figure 8a shows an interferogram of the oil-film contours by Wymer and Cameron [11] for a slender taper roller bearing in contact with an optically flat glass race under pure entraining motion. Taking the average taper radius of $0.0041 \mathrm{~m}$, a simulation run was undertaken for a cylindrical roller under the same operating conditions. The oil-film thickness contour obtained numerically is shown in Fig. 8b. A good qualitative comparison is observed between the numerical predictions and the experimental findings, although a quantitative comparison is inappropriate as the roller geometry only approximates the taper roller bearing 


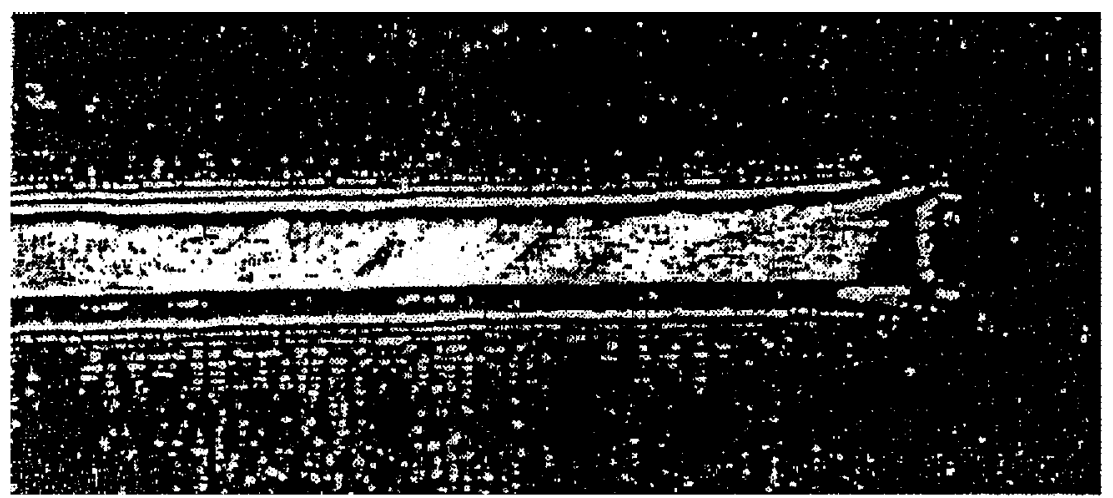

(a)

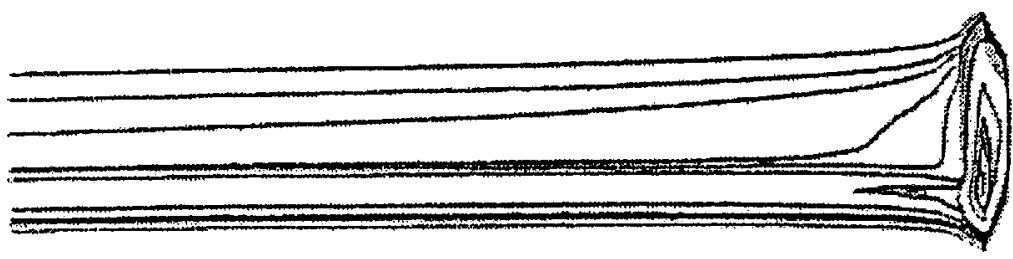

(b)

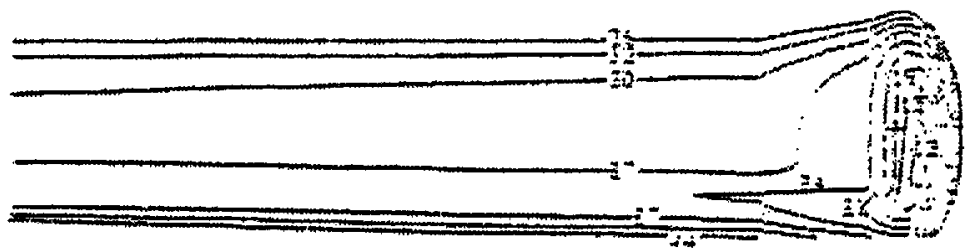

(c)

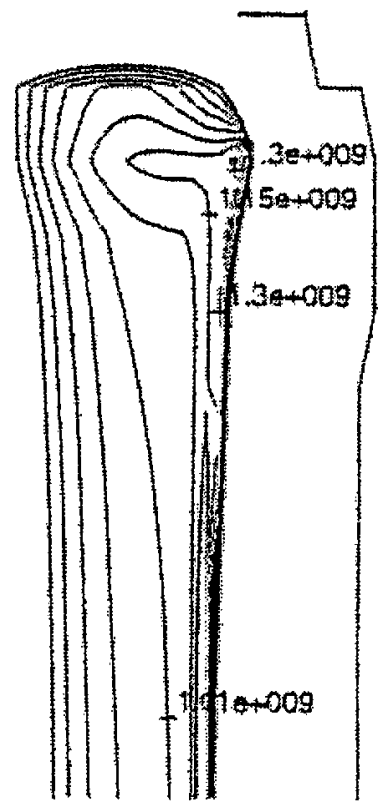

(d)

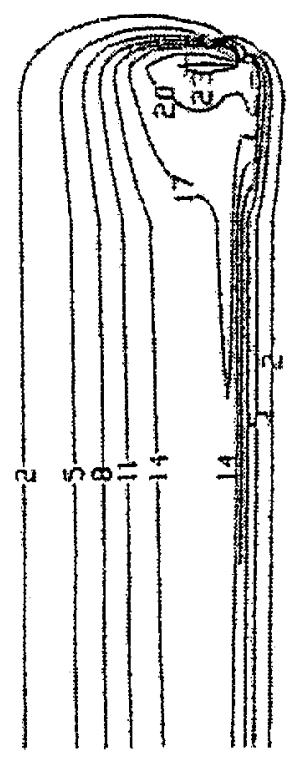

(e)

Fig. 8 (a) Interferogram by Wymer and Cameron [11]. (b) Oil-film contour obtained numerically. (c) Oil-film contour by Park and Kim [13]. (d) Pressure isobar for the Wymer and Cameron condition. (e) Pressure isobar by Park and Kim for the Wymer and Cameron condition 
used in the experiment. Park and Kim [13] have also provided a qualitative comparison with the same experimental results. Their oil-film contour is also shown in the same figure (Fig. 8c). Good agreement can be seen between all these findings. However, Park and Kim [13] have employed a computational grid of $56 \times 24$ elements, with the latter in the axial direction having irregular spacing according to an arithmetic progression, similar to that originally employed by Mostofi and Gohar [12]. In the present analysis, a regular mesh of $84 \times 162$ elements has been employed. The pressure isobars obtained here and by Park and Kim [13] for the aforementioned conditions are shown in Figs 8d and e.

Another comparison is made with the experimental work of Wymer and Cameron [11], this time at a higher speed of entraining motion at $4 \mathrm{~m} / \mathrm{s}$. Figures $9 a$ and $b$ show the experimental interferogram and the corresponding numerically obtained oil-film contours respectively. Good agreement is again observed under these conditions. Wymer and Cameron [11] have in fact carried out extensive measurements of lubricant film thickness for various values of entraining motion for blended and unblended rollers. Figure 10a shows a comparison of the current numerical predictions with their experimental results, represented by their extra- polated oil-film thickness equation found by regressional analysis of their results. In this figure, the numerical predictions given by Dowson and Higginson [9] extrapolated the oil-film thickness equation and these for a finite line contact given by Mostofi [23] have also been plotted. Good agreement is observed between all these findings. The power index for the variation of central film thickness with $U^{*}$ is between 0.64 and 0.67 . Figure $10 \mathrm{~b}$ shows the same comparison for the minimum exit film thickness in the direction of entraining motion and along the section 1-1 in the guide contour. Very good agreement is obtained between the numerical predictions here and the extrapolated oil-film thickness equation of Dowson and Higginson [9] for the minimum exit film. Wymer and Cameron's [11] obtained minimum exit film thickness formulae predicts thinner films, but with the same slope of variation with $U^{*}$, this being approximately 0.7 . The absolute minimum film thickness occurs in the end closure region to the side and the rear of the exit, as previously discussed. This should be the determining factor in the wear performance of the concentrated finite line contact.

Johns and Gohar [4] have shown that, in practice, under the loading condition described in Section 4, the bottom roller in a roller bearing is in fact subjected to a combination of compressive and moment loads,

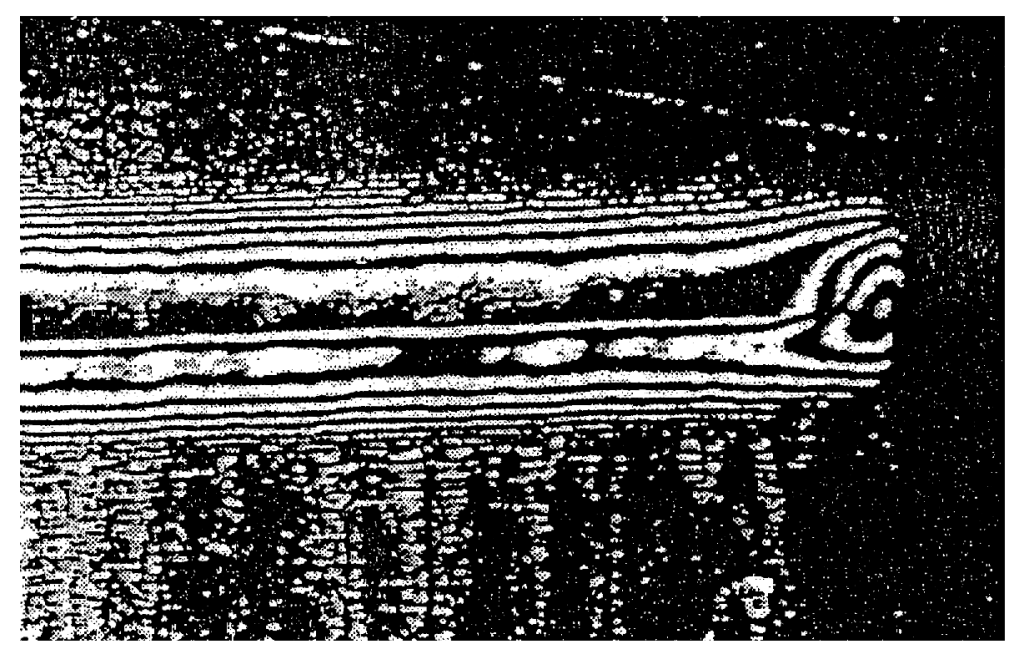

(a)

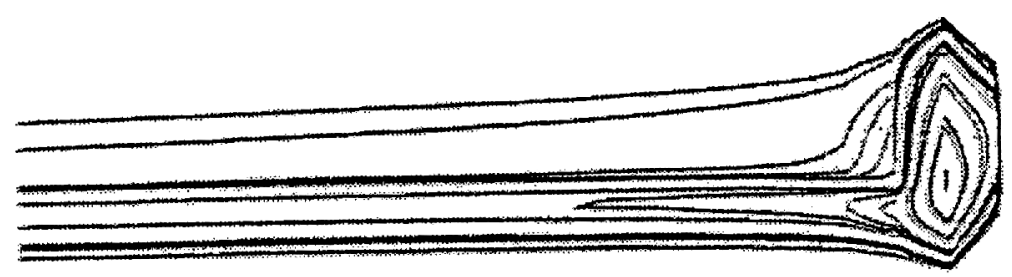

(b)

Fig. 9 (a) Interferogram by Wymer and Cameron [11] for high entraining speeds. (b) Oil-film contour obtained numerically 


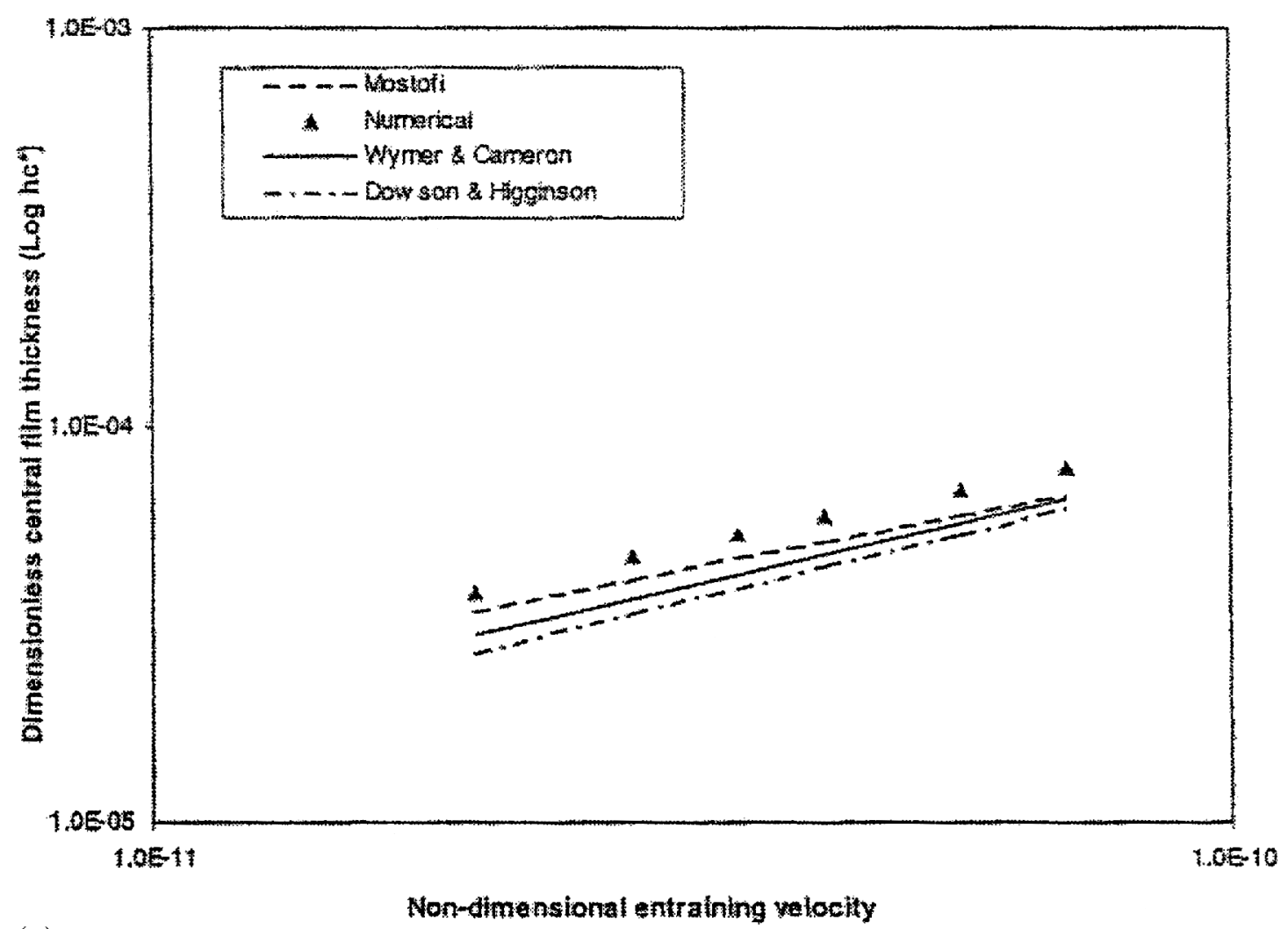

(a)

Non-dimeasionat entraining velocky

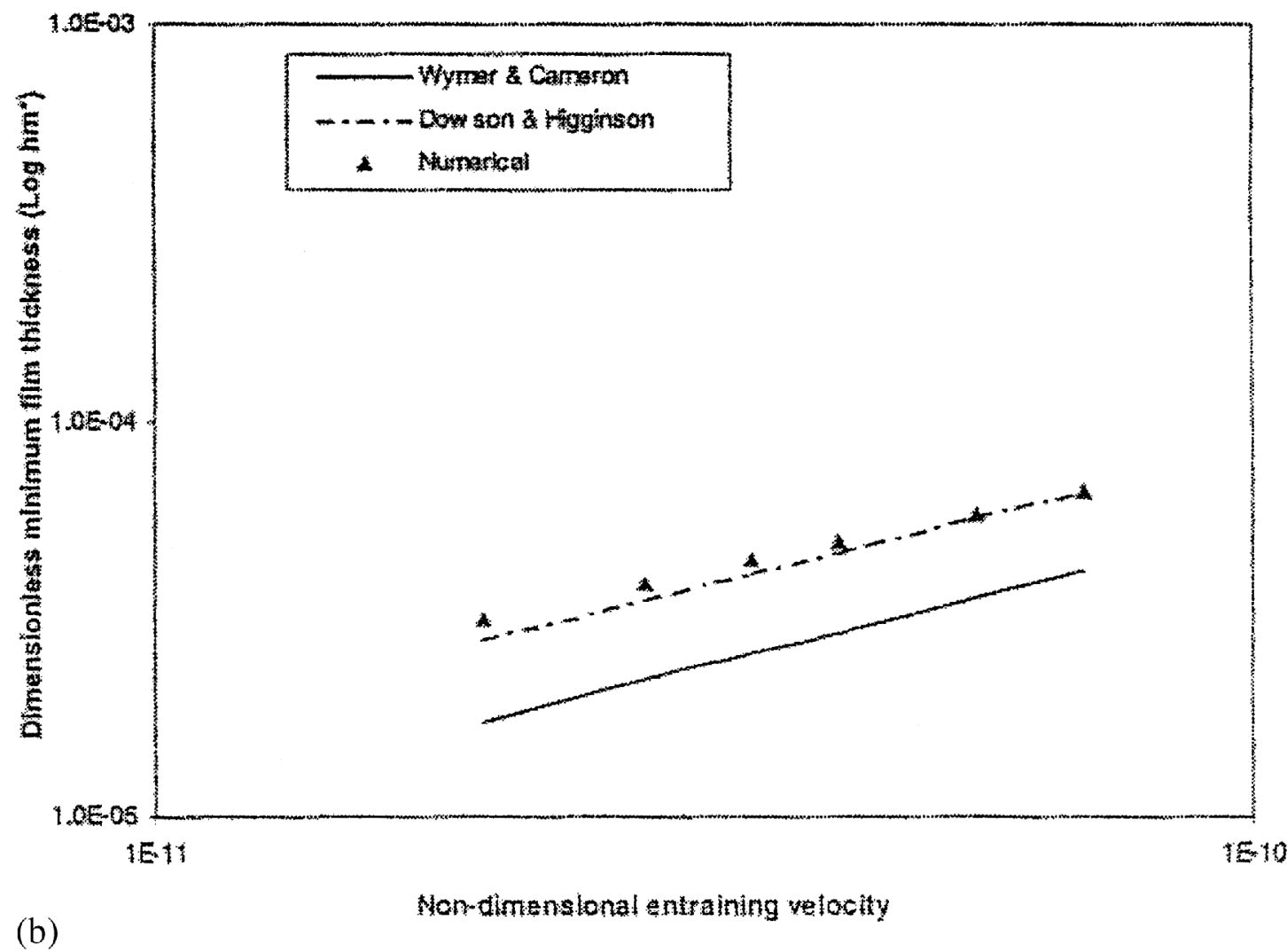

Fig. 10 (a) Variation of central film thickness with $\bar{U}$. (b) Variation of minimum exit film thickness with $\bar{U}$ 


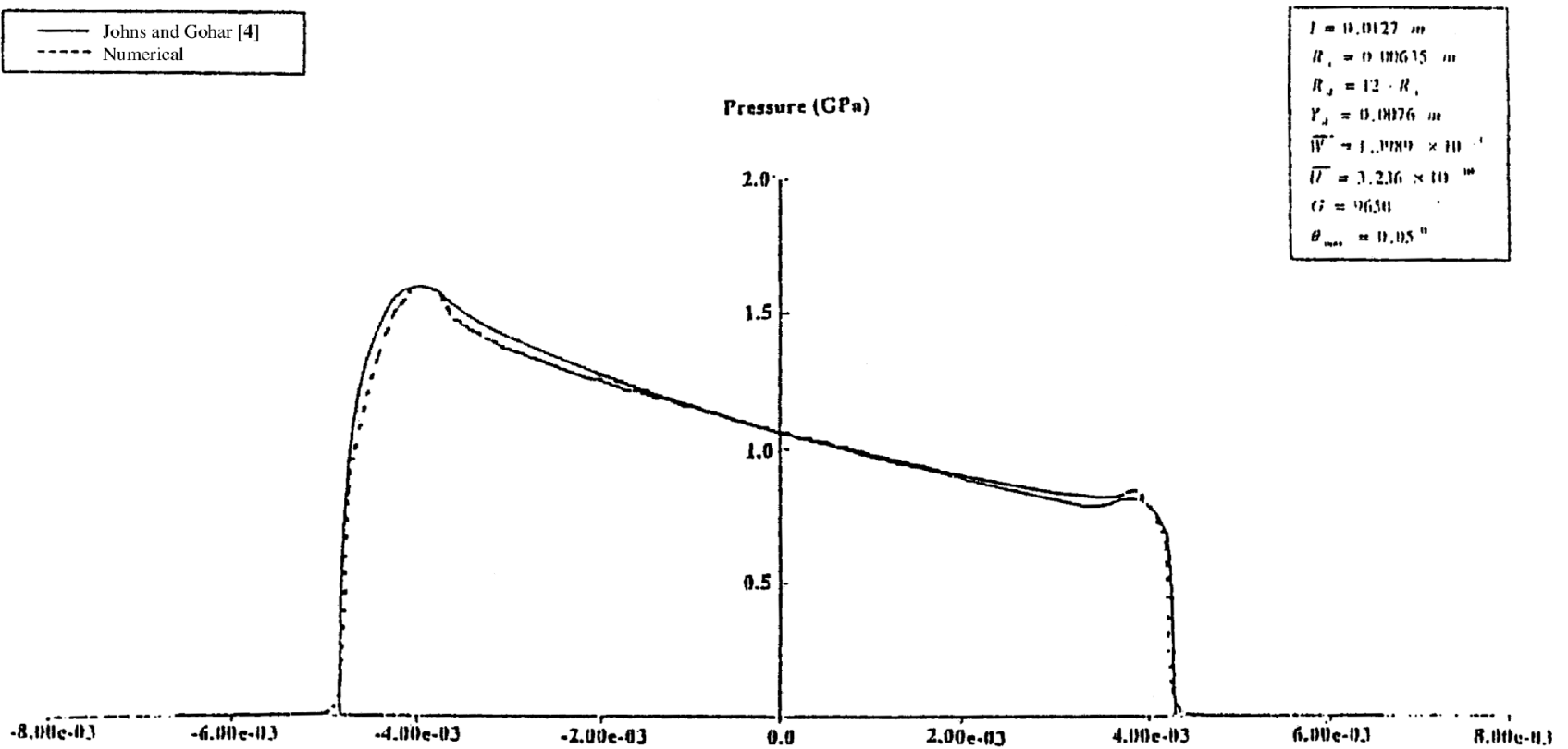

Transverse direction $Y(m)$

(a)

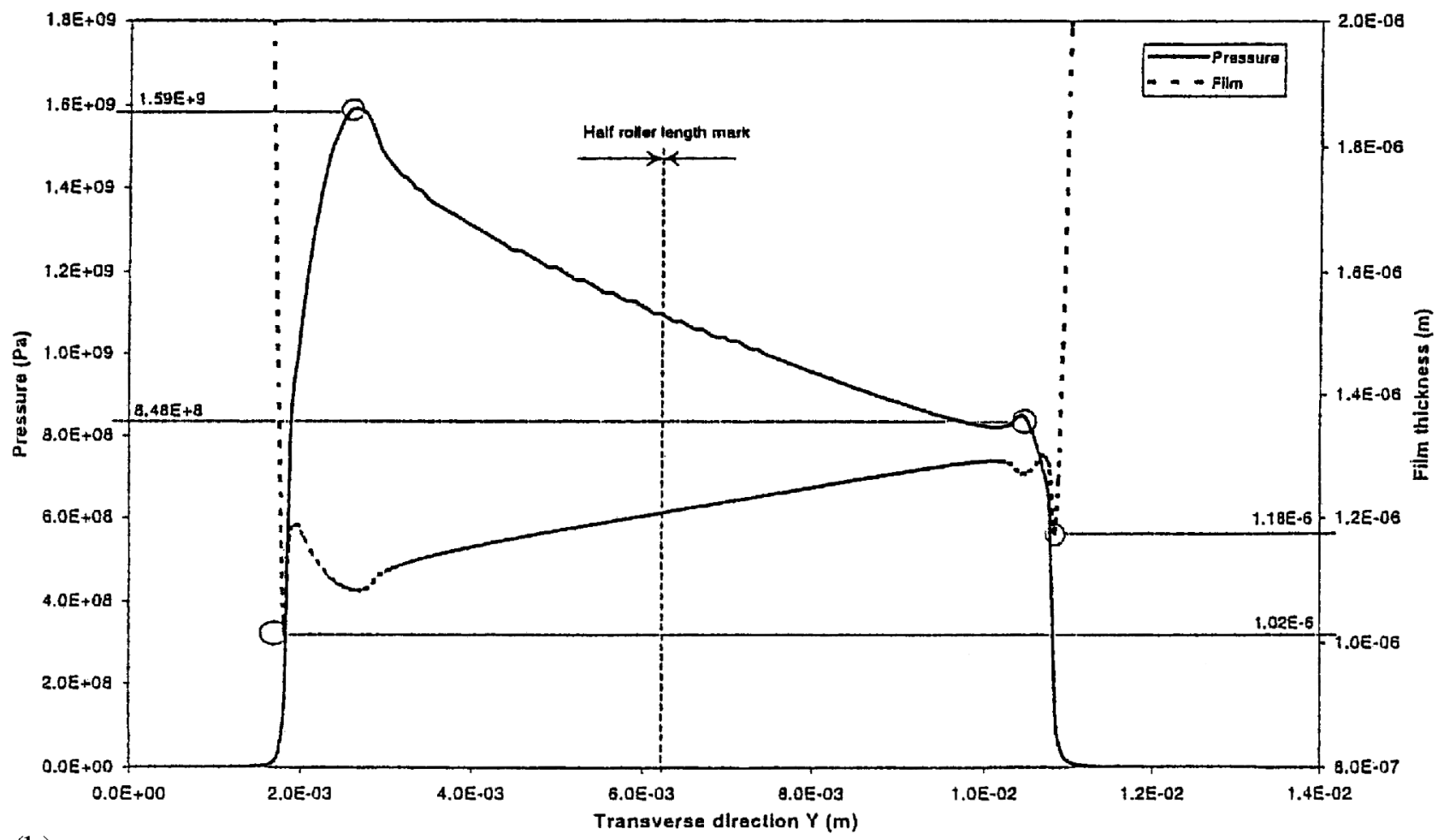

(b)

Fig. 11 (continued over)

resulting in a misalignment. Figure 11a shows the two central transverse pressure profiles for the same conditions as in Fig. 7a, but with a misalignment of angle $0.05^{\circ}$. One of the pressure profiles is taken from Johns and
Gohar [4] under elastostatic dry conditions, while the other is under the lubricated condition obtained in this analysis. Again, a close conformance is observed. The lubricated pressure profile in this figure is again shown in 

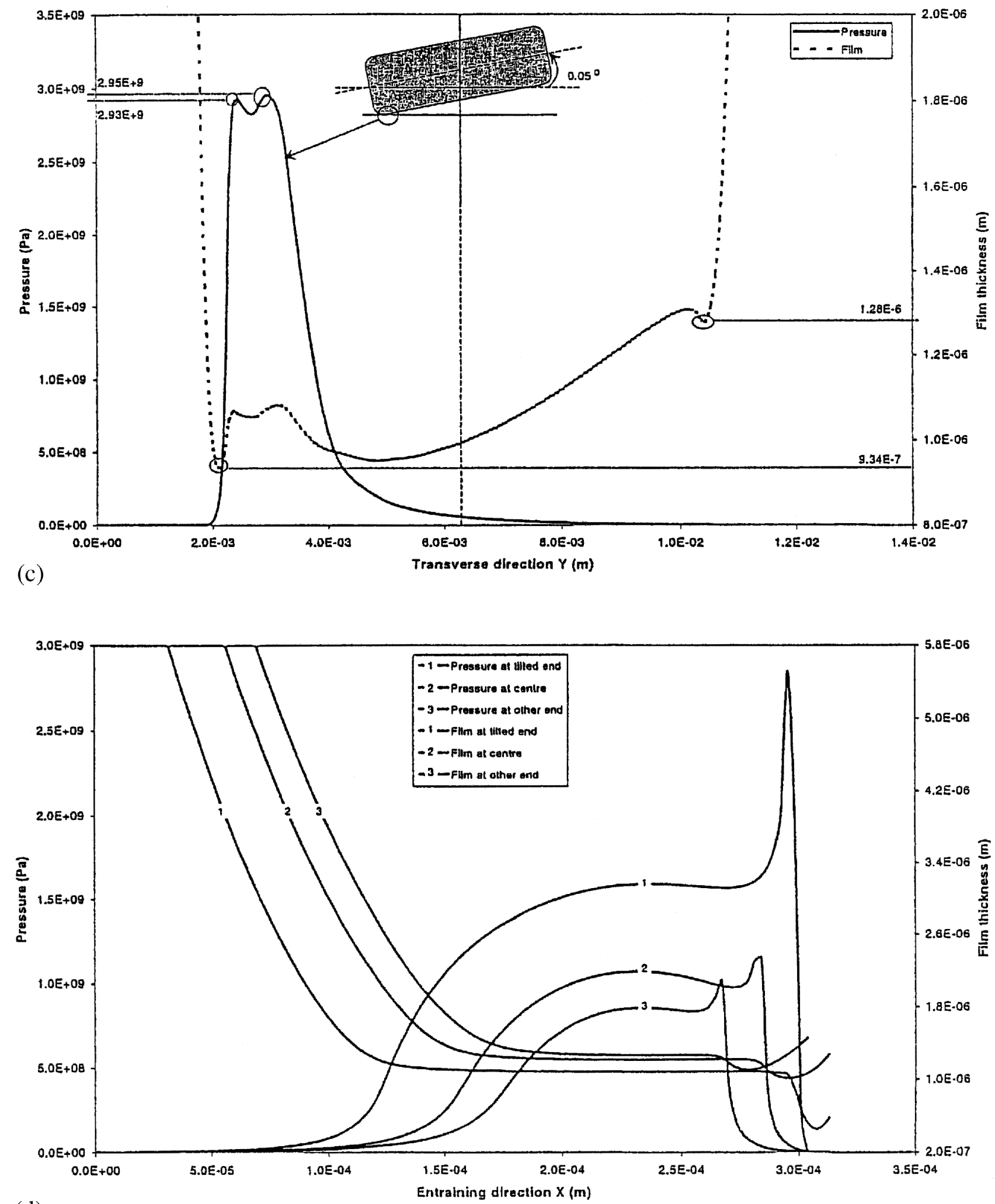

(d)

Fig. 11 (continued over)

Fig. 11b together with the corresponding central transverse section's oil-film shape. Comparing the minimum film thickness regions at the sides of the contact along this section with those of the aligned roller in Fig. 7c, it can be observed that the minimum film thickness at the loaded (i.e. tilted) end is hardly altered. In fact, the 

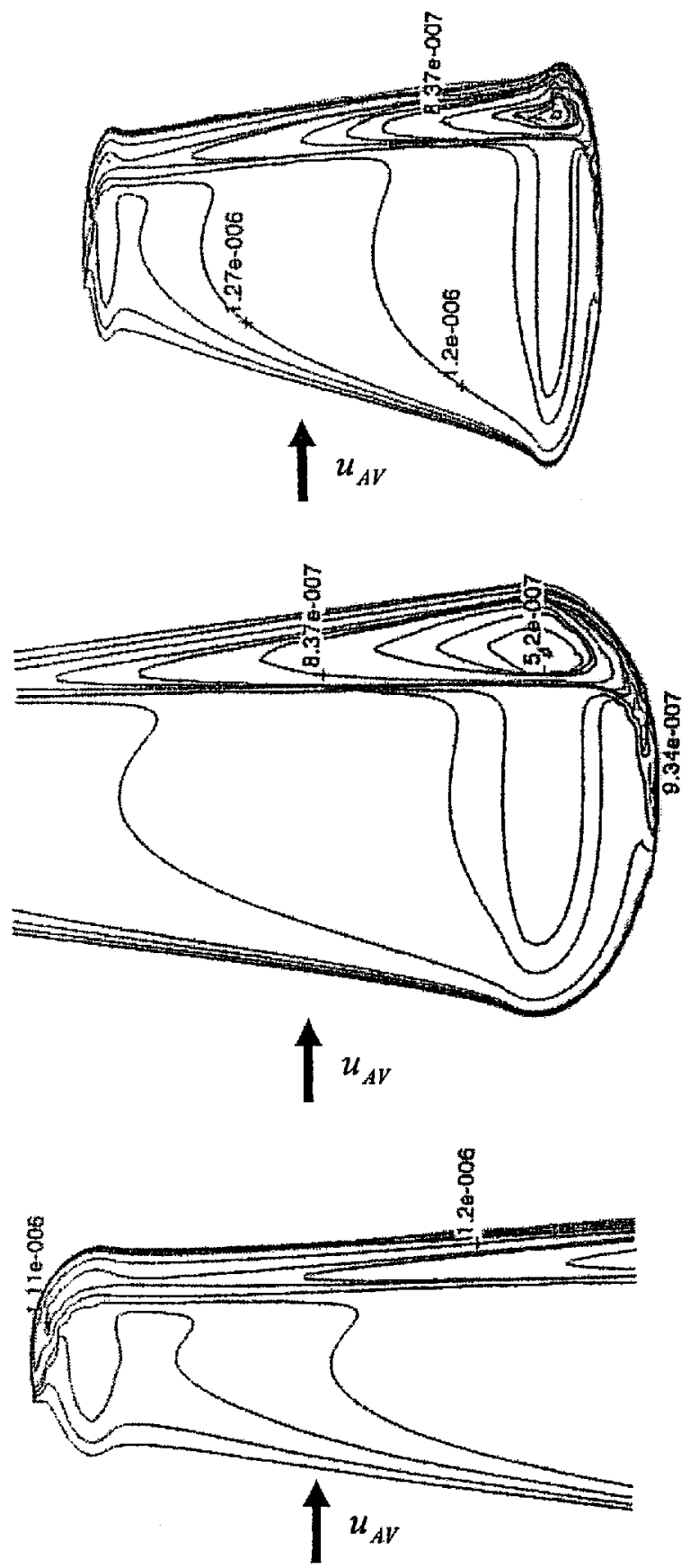

(e)

Fig. 11 (continued over)

oil-film thickness is mainly affected in the side constriction and in the exit region by the effect of misalignment. This fact is observed later on. The pressure profile through the side constriction (along section 4-4 in the guide contour) is shown in Fig. 11c. Due to the large misalignment, the pressure profile has become grossly asymmetrical, with large pressures generated only in the tilted direction. The maximum pressures have nearly reached a value of $3 \mathrm{GPa}$. These represent the severe conditions due to moment loading. The pressure spikes under such conditions converge to form a narrow region of high pressure, as shown by the twin peaks in the figure. The same figure shows the corresponding film thickness with a minimum value of $0.943 \mu \mathrm{m}$. Comparing this figure with that for the aligned roller (Fig. 7c), it can be noted that the minimum film thickness along this section has decreased in the tilted direction by approximately 9 per cent, while in the unloaded end of 

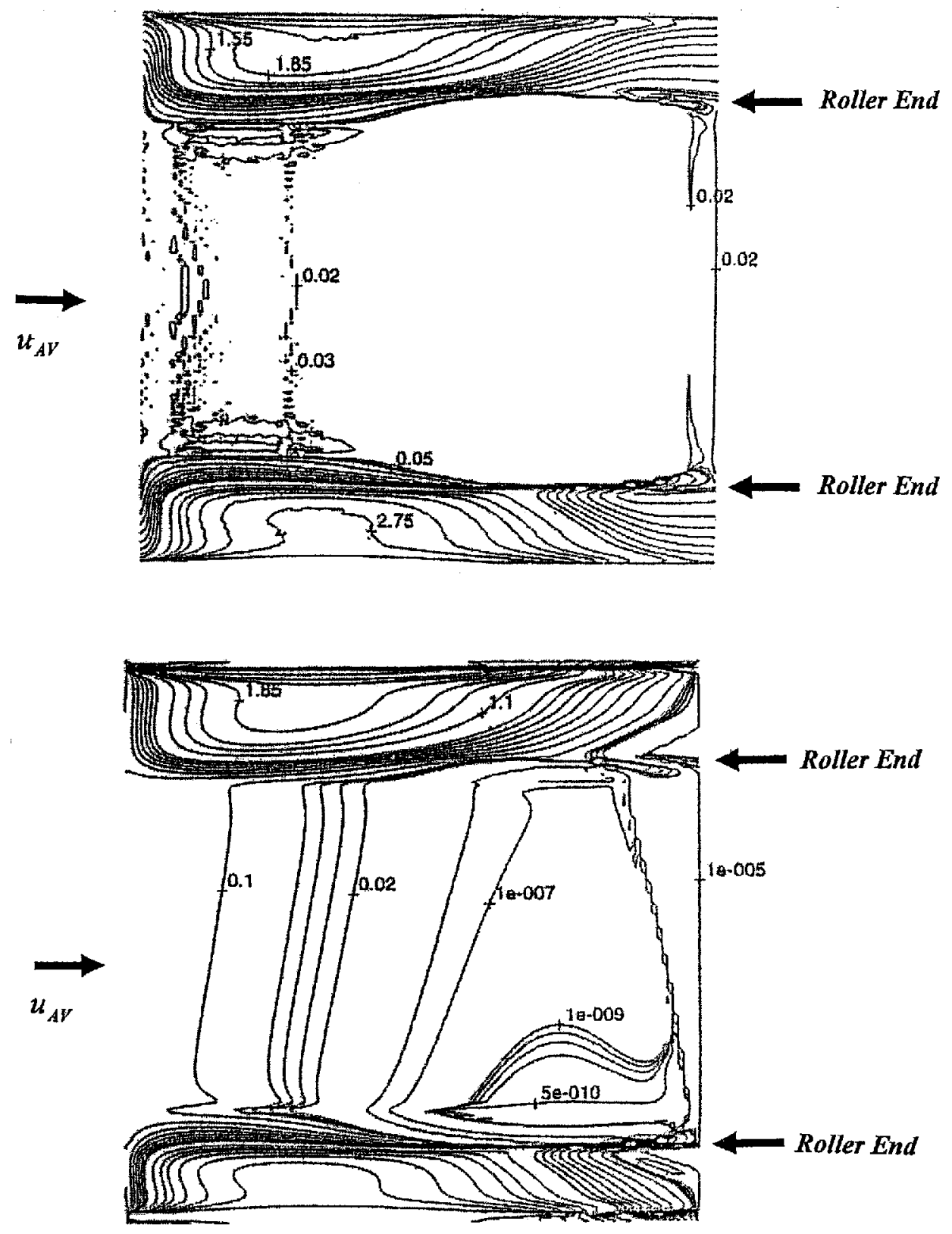

(f)

Fig. 11 (a) Central pressure profiles for the tilted roller along section 3-3. (b) Central pressure and corresponding film profiles along section 3-3. (c) Axial pressure and corresponding film profiles for the tilted roller along section 4-4. (d) Corresponding pressure and film profiles for the tilted roller at lifted, tilted and central points. (e) Oil-film contours for the misaligned roller (complete contours, tilted and lifted ends). (f) Oil-flow patterns for the roller aligned and misaligned ends

the roller, the gap has increased by approximately 28 per cent. The two-dimensional pressure cuts and the corresponding oil-film shapes in the direction of entraining motion through the centre of contact at the tilted end and at the other (lifted) end are shown in Fig. 11d. The absolute minimum oil-film thickness in the side constriction and at the rear of the contact in this figure is $0.455 \mu \mathrm{m}$. Note that the results in Fig. 11c are not through the corresponding absolute minimum film, but through a close-by section capturing the maximum pressure spike in the contact domain. Figure 11e shows the complete oil-film contour. The minimum oil-film thickness occurs in the tilted loaded region to the side and the rear of the contact (these are shown by the 
islands of minimum oil-film thickness in the figure). Note that the minimum oil-film thickness of $0.455 \mu \mathrm{m}$ occurs in the side constriction region of the tilted end and in the vicinity of the rear exit.

A good comparison between the aligned and misaligned contact conditions can be observed by the lubricant flow pattern through the contact. Figure $11 \mathrm{f}$ shows the flow pattern through the contact under an aligned roller contact with an applied load of $2256.6 \mathrm{~N}$ and the speed of entraining motion of $10 \mathrm{~m} / \mathrm{s}$. The flow pattern is obtained at each location in the computational zone as

$$
\bar{Q}=\sqrt{\bar{Q}_{x}^{2}+\bar{Q}_{y}^{2}}
$$

where $\bar{Q}_{x}$ is the non-dimensional flow in the direction of entraining motion and is given by

$$
\bar{Q}_{x}=\frac{u_{\mathrm{AV} \eta_{0} R^{2}}}{b^{3} P_{\mathrm{h}}}\left(\overline { \rho } \overline { h } \left\lceil-\frac{1}{12}\left(\frac{\bar{\rho} \bar{h}^{3}}{\bar{\eta}} \frac{\partial \bar{P}}{\partial \bar{x}}\right)\right.\right.
$$

and $\bar{Q}_{y}$ is the side flow in the axial direction (along the length of the roller) and is due to the pressure gradient only:

$$
\bar{Q}_{y}=-\frac{1}{12} \frac{b^{2}}{a^{2}}\left(\frac{\bar{\rho} \bar{h}^{3}}{\bar{\eta}} \frac{\partial \bar{P}}{\partial \bar{y}}\right)
$$

A feature of the elastohydrodynamic regime of lubrication is the flat film shape in the contact domain. This is corroborated by the low values of $\bar{Q}$ in the figure. The lubricant entrainment in the contact region has a much lower flowrate than that on the shoulders of the contact, shown in the figure by the arrows that indicate roller ends. The lubricant takes the path of least resistance. This means that the high pressures on the exit side and to the sides of the contact inhibit the inward flow of lubricant, which is induced by the entraining action. Therefore, fluid flow near these high-pressure regions has a swirl characteristic, as can be seen in the figure. These flow patterns give rise to the islands of minimum side constriction films that were observed in the oil-film contours of Fig. 11e. For the misaligned contact the asymmetry of pressure distribution disrupts the regular flow pattern. Many smaller swirl flow patterns emerge to the sides and rear exit of the contact domain, owing to the multiplicity of pressure peaks. Mostofi and Gohar [12] have referred to the generation of secondary pressure peaks as being possibly the result of an inward diffusion of lubricant due to Poiseuille flow into these regions. This would explain the higher elastohydrodynamic pressure spikes in these regions than those predicted under dry elastostatic conditions. The results shown in Fig. 11f give 'some' credence to their observations.

\section{CONCLUSIONS}

The results show that the prevailing conditions at the contact extremities and in the direction transverse to that of entraining motion often represent the most severe cases, both in terms of generated pressures and lubricant film thickness. The pressure spikes clearly cause secondary subsurface stress fields, as shown by Johns-Rahnejat and Gohar [25], thus inducing fatigue spalls. The corresponding absolute minimum film thickness occurs in the vicinity of the pressure spikes and would be the deciding factor for the onset of wear. Thus, the justification for a finite line contact solution for the case of roller-to-race and cam-and-follower pairs is evident.

\section{REFERENCES}

1 Harris, T. A. The effect of misalignment on the fatigue life of cylindrical roller bearings having crowned rolling members. Trans. ASME, J. Lubric. Technol., 1969, 91.

2 Gohar, R. A numerical method for obtaining the deformed shape of the roll in the cold rolling process. Proc. Instn Mech. Engrs, Part C, Journal of Mechanical Engineering Science, 1974, 16.

3 Heydari, M. and Gohar, R. Pressure distribution on radially loaded rollers. Proc. Instn Mech. Engrs, Part C, Journal of Mechanical Engineering Science, 1979, 16.

4 Johns, P. M. and Gohar, R. Roller bearings under radial and eccentric loads. Tribology Int., June 1981, 13.

5 Rahnejat, H. and Gohar, R. Design of profiled tapered roller bearing. Tribology Int., December 1979, 11.

6 Hartnett, M. The analysis of contact stresses in rolling element bearings. Trans. ASME, J. Lubric. Technol., 1979, 101.

7 Kannel, J. W. Comparison between predicted and measured axial pressure distribution between cylinders. Trans. A SME, J. Lubric. Technol., 1974, 96.

8 Zantapulos, H. The effect of misalignment on the fatigue life of tapered roller bearings. Trans. ASME, J. Lubric. Technol., April 1972, 94.

9 Dowson, D. and Higginson, G. R. Elastohydrodynamic Lubrication, 1966 (Pergamon Press, New York).

10 Niemann, G. and Gartner, F. Distribution of hydrodynamic pressure on counterformal line contacts. Trans. ASLE, $1965,8(3)$.

11 Wymer, D. G. and Cameron, A. EHL lubrication of a line contact. Part 1: optical analysis of a roller bearing. Proc. Instn Mech. Engrs, 1973-4, paper 18, 188.

12 Mostofi, A. and Gohar, R. Elastohydrodynamic lubrication of finite line contacts. Trans. ASME, J. Lubric. Technol., 1983, 105, 598-604.

13 Park, T. J. and Kim, K. W. Elastohydrodynamic lubrication of a finite line contact. Wear (Elsevier Science), 1998, 223, 102-109.

14 Ehret, P., Dowson, D., Taylor, C. M. and Wang, D. Analysis of isothermal elastohydrodynamic point contacts lubricated by Newtonian fluids using multigrid methods. 
Proc. Instn Mech. Engrs, Part C, Journal of Mechanical Engineering Science, 1997, 211(C7), 493-508.

15 Jalali-Vahid, D., Rahnejat, H., Gohar, R. and Jin, Z. M. Prediction of oil film thickness and shape in elliptical point contacts under combined rolling and sliding motion. Proc. Instn Mech. Engrs, Part J, Journal of Engineering Tribology, 2000, 214(J5), 427-437.

16 Rahnejat, H. Influence of vibration on oil film in concentrated contacts. $\mathrm{PhD}$ thesis, Imperial College of Science and Technology, University of London, 1984.

17 Roelands, C. J. A. Correlation aspects of viscositytemperature-pressure relationship of lubricating oils. PhD thesis, Delft University of Technology, The Netherlands, 1966.

18 Johnson, K. L. Contact Mechanics, 1985 (Cambridge University Press).

19 Johns, P. M. The design of cylindrical rollers for use in shaft and bearing systems. MSc thesis, Imperial College of Science and Technology, University of London, 1978.

20 Harris, T. A. Rolling Bearing Analysis, 1966 (John Wiley, New York).
21 Lundberg, G. Elastic contact between two semi-infinite bodies. Forschung auf dem Gebieto des Engenieuswesens, 1961, 10(5), 165-174.

22 Moyer, C. A. and Neifert, H. R. A first order solution for the stress concentration present at the end of roller contact. ASLE Trans., 1963, 6, 324-336.

23 Mostofi, A. Oil film thickness and pressure distribution in elastohydrodynamic elliptical contacts. PhD thesis, Imperial College of Science and Technology, University of London, 1981.

24 Grubin, A. N. and Vinogradova, I. E. Investigation of Scientific and Industrial Research, Book 30, 1949 (Central Scientific Research Institute for Technology and Mechanical Engineering, Moscow).

25 Johns-Rahnejat, P. M. and Gohar, R. Point contact elastohydrodynamic pressure distribution and sub-surface stress field. In Tri-Annual Conference on Multi-Body Dynamics: Monitoring and Simulation Techniques (Eds H. Rahnejat and R. Whalley), 1997 (Mechanical Engineering Publications, London). 